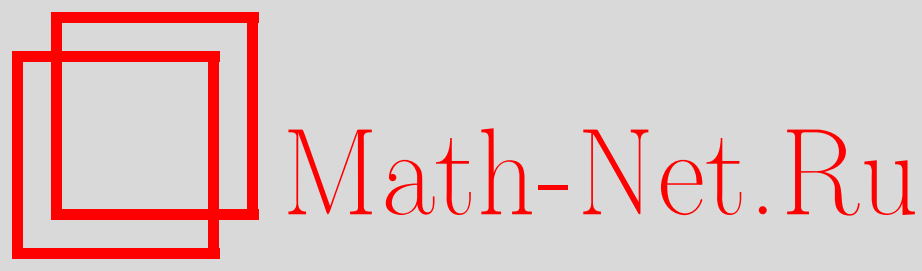

В. Р. Фаталов, Ряды теории возмущений в квантовой механике: фазовые переходы и точные асимптотики для коэффициентов разложения, ТМФ, 2013, том 174, номер 3, 416-443

DOI: https://doi.org/10.4213/tmf8326

Использование Общероссийского математического портала Math-Net.Ru подразумевает, что вы прочитали и согласны с пользовательским соглашением http://www . mathnet.ru/rus/agreement

Параметры загрузки:

IP : 54.209 .52 .79

26 апреля 2023 г., 16:49:35

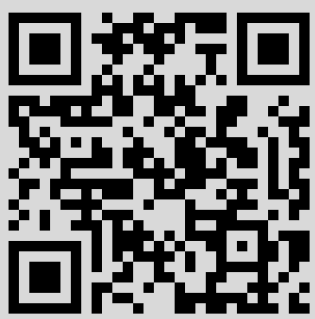




\title{
В. Р. Фаталов* \\ РЯДЫ ТЕОРИИ ВОЗМУЩЕНИЙ В КВАНТОВОЙ МЕХАНИКЕ: ФАЗОВЫЕ ПЕРЕХОДЫ И ТОЧНЫЕ АСИМПТОТИКИ ДЛЯ КОЭФФИЦИЕНТОВ РАЗЛОЖЕНИЯ
}

\begin{abstract}
Рассматривается модель гармонического осциллятора со степенным потенциалом. Получены новые асимптотические формулы для коэффициентов разложения рядов теории возмущений по степеням константы связи в случае возмущения посредством степенного потенциала $|x|^{p}, p>0$. Доказано существование и вычислено критическое значение $p_{0}$, которое является пороговым в том смысле, что исследуемые коэффициенты имеют качественно разные асимптотики при $0<p<p_{0}$ и при $p>p_{0}$. Указано, что при $p=p_{0}$ в рассматриваемой физической системе происходит фазовый переход. Исследование проводится с помощью метода Лапласа для функциональных интегралов по гауссовским мерам.
\end{abstract}

Ключевые слова: фазовые переходы, ряды теории возмущений, формула следа Либа, условные винеровские меры, метод Лапласа в банаховом пространстве.

DOI: $10.4213 / \operatorname{tmf} 8326$

\section{1. ВВЕДЕНИЕ И ФОРМУЛИРОВКА ОСНОВНЫХ РЕЗУЛЬТАТОВ}

1.1. Типы фазовых переходов в теоретической физике и метод Лапласа для функциональных интегралов. Существует тесная идейная связь между теорией фазовых переходов в математической физике [1], [2] и методом Лапласа в функциональных пространствах [3], [4]. Эта связь основана на вариационном принципе Гиббса, который играет ключевую роль как в теории фазовых переходов, так и в методе Лапласа (см. [1]-[3]). Здесь можно выделить три возможные ситуации для проведения очевидных аналогий.

1. Рассматриваемый гладкий лагранжиан $\mathcal{L}$ в той или иной физической теории (квантовая механика, теория поля и другие) имеет единственное невырожденное основное состояние [1]-[5]. Основное состояние принадлежит множеству стационарных (критических, экстремальных) точек лагранжиана $\mathcal{L}$, невырожденность основного состояния означает невырожденность второй производной $\mathcal{L}^{\prime \prime}$ лагранжиана,

* Московский государственный университет им. М. В. Ломоносова, Москва, Россия. E-mail:vrfatalov@yandex.ru 
сам лагранжиан $\mathcal{L}$ часто называют действием. В различных физических теориях основное состояние носит такие названия, как равновесное состояние, гиббсовская мера, экстремальная мера и т. д. Метод Лапласа в функциональных пространствах, восходящий к Фейнману [6], позволяет выписать в данном случае точную асимптотику функционального интеграла, зависящего от большого параметра.

2. Особый интерес представляет случай, когда лагранжиан зависит от некоторого параметра $p>0, \mathcal{L}=\mathcal{L}(p)$. Пусть существует некоторое пороговое значение $p_{0}>0$ такое, что для значений $0<p \leqslant p_{0}$ лагранжиан $\mathcal{L}(p)$ имеет единственное невырожденное основное состояние, а для значений $p>p_{0}$ лагранжиан $\mathcal{L}(p)$ имеет многообразие невырожденных основных состояний (критических) точек. Тогда мы будем говорить, что при значении параметра $p=p_{0}$ происходит фазовый переход nервого типа. В этом случае точная асимптотика упомянутого выше функционального интеграла имеет иной вид по сравнению со случаем 1 (см. теорему 3.3 в [3] и работу [7]).

3. Возможен также случай, когда для некоторого порогового значения $p_{0}>0$ лагранжиан $\mathcal{L}(p)$ имеет единственное невырожденное основное состояние при значениях $0<p \leqslant p_{0}$, а при $p>p_{0}$ - единственное вырожденное основное состояние. Тогда мы будем говорить, что при значении параметра $p=p_{0}$ происходит фазовый переход второго тuпа. В этом случае точная асимптотика упомянутого выше функционального интеграла также имеет иной вид по сравнению со случаем 1 (см. теорему 3.2 в [3]).

Из этих рассуждений следует важный вывод. Возможны ситуации, когда исследование точных асимптотик функциональных интегралов типа Лапласа в той или иной параметрической физической модели позволяет сделать вывод о существовании фазового перехода первого или второго типа и найти пороговое значение $p_{0}$.

Один пример такой ситуации рассмотрен и исследован в статье [4]. В настоящей работе мы рассмотрим второй пример такого рода. Отметим, что техника, развитая в работах [3], [4], [7]-[12], позволяет решать многие экстремальные задачи, связанные с методом Лапласа и фазовыми переходами, для (много)параметрических физических моделей, которые основаны на гауссовских мерах, условных винеровских мерах, а также для распределений диффузионных марковских процессов и сумм независимых одинаково распределенных случайных элементов.

1.2. Формулировка основных результатов. Метод функционального (потраекторного) интегрирования находит широкое и эффективное применение в таких разделах современной теоретической физики, как квантовая теория поля, статистическая механика и ряде других [1], [13]-[15]. Особый интерес представляет важный и распространенный в теории и приложениях случай, когда исследуемый функциональный (континуальный) интеграл берется по некоторой гауссовской (в частности, условной винеровской) мере в пространстве непрерывных функций. Дело в том что в случае гауссовского функционального интеграла можно провести многие необходимые вычисления, используя хорошо развитые приближенные и асимптотические методы теории гауссовских мер [3], [8], [16]-[18]. В настоящей статье мы исследуем один важный и интересный пример подобного рода, связанный с рядами теории возмущений [13], [19]-[32].

Рассмотрим обобщение задачи теории возмущений, придерживаясь изложения книги [13]. 
Пусть $L^{2}=L^{2}(\mathbb{R})$ - обычное гильбертово пространство действительных функций, заданных на оси $\mathbb{R}$, со скалярным произведением $\langle y, z\rangle=\int_{-\infty}^{\infty} y(x) z(x) d x$. Пусть

$$
L_{0}:=-\frac{1}{2} \frac{d^{2}}{d x^{2}}+\frac{x^{2}}{2}-\frac{1}{2}
$$

- линейный дифференциальный оператор в $L^{2}$, заданный на множестве $\mathcal{D}$ дважды дифференцируемых функций $y \in L^{2}$ таких, что $L_{0} y \in L^{2}$ [13]. Данный оператор является существенно самосопряженным на $C_{0}^{\infty}(\mathbb{R})[5]$ и связан очевидным образом с гармоническим осциллятором $-d^{2} / d x^{2}+x^{2}$. Собственные числа и собственные функции гармонического осциллятора хорошо известны (см., например, дополнение к $\S$ V.3 в т. 1 книги [5] и доказательство теоремы 4.6 в [13]). Учитывая этот факт, мы приходим к выводу о положительности оператора $L_{0}$, таким образом, при всех $y \in \mathcal{D}$ выполнено неравенство $\left\langle L_{0} y, y\right\rangle \geqslant 0$.

Для фиксированных чисел $p>0$ и $g \geqslant 0$ рассмотрим следующее возмущение оператора $L_{0}$ посредством степенного потенциала:

$$
L_{0}(g):=L_{0}+g|x|^{p} \equiv-\frac{1}{2} \frac{d^{2}}{d x^{2}}+\frac{x^{2}}{2}-\frac{1}{2}+g|x|^{p},
$$

параметр $g$ называется константой связи.

Пусть $\mathcal{D}_{p}:=\left\{y(x) \in \mathcal{D}:|x|^{p} y(x) \in L^{2}\right\}$ - всюду плотное множество в $L^{2}$. Как указано в следующем предложении, оператор $L_{0}(g)$ обладает целым рядом хороших свойств.

ПреДЛОЖЕНИЕ 1.1. Оператор $L_{0}(g)$ muпа Шредингера при $g>0$ является строго положительным существенно самосопряженным оператором, таким образом, при всех $0 \neq y \in \mathcal{D}_{p}$ выполнено неравенство $\left\langle L_{0}(g) y, y\right\rangle>0$. Этот оператор имеет чисто дискретный простой спектр, а основная собственная функция (основное состояние) является строго положительной.

ДокАЗАтЕльство. Строгая положительность оператора $L_{0}(g)$, очевидно, следует из положительности оператора $L_{0}$. Утверждения о самосопряженности и спектре оператора $L_{0}(g)$ следуют из общих известных результатов (см., например, книгу [5], т. 4 и сборник [33]). Строгая положительность основной собственной функции следует из теоремы XIII.47 в т. 4 книги [5].

Обозначим через $E(g)>0$ энергию основного состояния, т. е. минимальное собственное число оператора $L_{0}(g)$ при $g>0$. Во многих физических задачах вычисление энергии основного состояния $E(g)$ - это важная и сложная проблема. Случаи $p=4$ и $p=2 N$ рассмотрены в [13] и [26] в связи с $\phi^{2 N}$-моделью теории поля (см. также т. 2 книги [5], § Х.7, и [24], [34]). В настоящей статье мы рассмотрим случай общего $p>0$, используя подход из книги [13].

Применяя методы теории возмущений [5], [35], мы ставим в соответствие собственному числу $E(g)$ формальный ряд теории возмущений:

$$
E(g) \simeq \sum_{n=0}^{\infty} a_{n} g^{n} .
$$

При малых $g$ этот ряд является асимптотическим в смысле Пуанкаре (см. [13], а также книгу [5], т. 4) и оказывается расходящимся (см., например, [31]). Тем не менее 
известно, что в различных моделях теории поля и квантовой механики при достаточно малых $g$ вычисления, проведенные с учетом нескольких первых членов ряда (1.2), дают хорошее согласие с экспериментальными данными и данными, полученными иными методами (например, вариационным методом или методом ренормгруппы). Типичной здесь является ситуация, описанная при $p=4$ в книге [5], т. 4 , § ХІІ.3 (см. также пример 3 в $\S$ XII.4, т. 4 книги [5] и статью [36]).

Непосредственное вычисление самих коэффициентов $a_{n}$ ряда (1.2) - непростая задача. Весьма эффективным и полезным средством при ее решении является формула Фейнмана-Каца, позволяющая связать ряд (1.2) с другим формальным асимптотическим рядом (см. формулу (1.7) ниже). Согласно формуле Фейнмана-Каца выполнено следующее соотношение [13]:

$$
E(g)=-\lim _{\beta \rightarrow \infty} \frac{1}{\beta} \ln \operatorname{Tr}\left(e^{-\beta L_{0}(g)}\right), \quad g>0
$$

где $\beta$ - обратная температура [26], $\operatorname{Tr} A$ обозначает след оператора $A$. Из формулы (1.3) следует, что для вычисления $E(g)$ нужно знать значения $\operatorname{Tr}\left(e^{-\beta L_{0}(g)}\right)$ при больших $\beta$. Для решения последней задачи часто применяется замечательная формула Либа, связывающая указанный след с функциональным интегралом по условной винеровской мере [13], [26], [37]. Мы запишем эту формулу в нашем случае, придерживаясь общепринятых обозначений теории случайных процессов.

Пусть $[\Omega, \mathcal{F}, \mathbf{P}]$ - основное вероятностное пространство, $\mathbf{E}$ - оператор математического ожидания, взятый относительно вероятности Р. Везде ниже $w(t), t \geqslant 0$, обозначает стандартный винеровский процесс, рассматриваемый как марковский процесс [38]-[40]. Ниже термин "распределение" используется как синоним термина "вероятностная мера".

Пусть $C[0, \beta]$ - банахово пространство непрерывных на $[0, \beta]$ действительных функций $x(t)$ с нормой $\|x\|_{\infty}:=\sup _{t \in[0, \beta]}|x(t)|$ и борелевской $\sigma$-алгеброй $\mathcal{B}_{\beta}$. Обозначим через $P_{w}\{(\cdot) \mid x(0)=y\}$ распределение в пространстве $C[0, \beta]$ винеровского процесса, выходящего из точки $y \in \mathbb{R}$. Таким образом, для любого $C \in \mathcal{B}_{\beta}$ выполнено равенство

$$
P_{w}\{C \mid x(0)=y\}=\mathbf{P}\{w \in C \mid w(0)=y\} .
$$

Аналогично для точек $y, z \in \mathbb{R}$ определим условное распределение в пространстве $C[0, \beta]$ винеровского процесса, выходящего в начальный момент из точки $y$ и приходящего в момент $\beta$ в точку $z$ :

$$
P_{w}\{C \mid x(0)=y, x(\beta)=z\}=\mathbf{P}\{w \in C \mid w(0)=y, w(\beta)=z\}
$$

для любого $C \in \mathcal{B}_{\beta}$. Отметим, что распределение (1.4) при $y=0$, т. е. распределение $P_{w}\{(\cdot) \mid x(0)=0\}$, общепринято называть винеровской мерой в $C[0, \beta]$ (см., например, [41]). Отметим, что в интересной и содержательной книге [13] винеровской мерой назван иной объект, и это надо иметь в виду при чтении книги [13]. Распределение (1.5) часто называют условной винеровской мерой, а также распределением броуновского моста из точки у в точку z [39]. Во введенных обозначениях упомянутая формула Либа для следа записывается следующим образом. 
ПреДЛОЖЕНИЕ 1.2. Для чисел $p>0, g \geqslant 0, \beta>0$ u оператора $L_{0}(g)$, заданного в (1.1), справедливо равенство

$$
\begin{aligned}
\operatorname{Tr}\left(e^{-\beta L_{0}(g)}\right)= & \frac{e^{\beta / 2}}{\sqrt{2 \pi \beta}} \int_{-\infty}^{\infty} d y \int_{C[0, \beta]} \exp \left\{-g \int_{0}^{\beta}|x(t)|^{p} d t-\frac{1}{2} \int_{0}^{\beta} x^{2}(t) d t\right\} \times \\
& \times P_{w}\{d x \mid x(0)=x(\beta)=y\} .
\end{aligned}
$$

Формула (1.6), отсутствующая в книге [13], получена нами на основе соотношений $(9.6),(18.5),(18.6)$ из [13], при этом мы выразили меры $d \mu_{0, T}, d \mu_{0, x, x, t}$ с помощью распределения (1.5). Формулу (1.6) можно проверить при $g=0$ : вычисляя двумя разными способами при $g=0$ левую и правую части равенства (1.6), мы получаем одно и то же число $e^{\beta} /\left(e^{\beta}-1\right)$. Вычисление левой части равенства (1.6) при $g=0$ проводится просто, следует рассмотреть известные значения всех собственных чисел оператора $L_{0}$, описанные в книге [5], т. 1. Вычисление правой части равенства (1.6) при $g=0$ проводится значительно сложнее, оно основано на формуле (3.18) из [13] для гауссовских интегралов типа Лапласа с квадратичной функцией в показателе экспоненты.

Разлагая экспоненту в правой части формулы (1.6) в ряд по степеням константы связи $g$ и формально меняя порядок суммирования и интегрирования, мы получаем следующее формальное равенство:

$$
\operatorname{Tr}\left(e^{-\beta L_{0}(g)}\right) \simeq \sum_{n=0}^{\infty} b_{n}(\beta) g^{n},
$$

где

$$
\begin{aligned}
& b_{n}(\beta) \equiv b_{n}(\beta, p)=\frac{(-1)^{n} e^{\beta / 2}}{n ! \sqrt{2 \pi \beta}} \int_{-\infty}^{\infty} d y \int_{C[0, \beta]}\left(\int_{0}^{\beta}|x(t)|^{p} d t\right)^{n} \times \\
& \times \exp \left\{-\frac{1}{2} \int_{0}^{\beta} x^{2}(t) d t\right\} P_{w}\{d x \mid x(0)=x(\beta)=y\} .
\end{aligned}
$$

Ряд (1.7) для малых $g$ является асимптотическим в смысле Пуанкаре и также оказывается расходящимся. Как указано в книге [13], связь между двумя рядами (1.2) и (1.7) несложная, следует учесть формулы (1.3), (1.6) и разложение Тейлора для функции $\ln (1+x)$. Информация о коэффициентах $b_{n}(\beta)$ при больших фиксированных $\beta$ используется для получения оценок и приближенных значений коэффициентов $a_{n}$ (см., в частности, формулы (7)-(9) в статье [26]). Большой интерес представляет порядок роста коэффициентов $a_{n}$ и $b_{n}(\beta)$ при $n \rightarrow \infty$ (см. работы [19]-[22], [25]-[28], литературные ссылки в [31], [32], а также теорему 18.3 в [13]).

В настоящей статье на основе метода Лапласа в функциональном пространстве, примененного к интегралу в (1.8), мы получим теорему о точной асимптотике коэффициентов $b_{n}(\beta, p)$ при $n \rightarrow \infty$ и фиксированных $p>0, \beta>0$. Насколько известно автору, в литературе подобная задача не рассматривалась. Особый интерес в физических теориях представляет случай $p=2 N$, где натуральный параметр $N$ - размерность. Оказалось, что в указанной выше задаче существует пороговое значение

$$
p_{0} \equiv p_{0}(\beta):=2+\frac{4 \pi^{2}}{\beta^{2}}
$$

в том смысле, что коэффициенты $b_{n}(\beta, p)$ имеют качественно разные асимптотики при $0<p<p_{0}$ и при $p>p_{0}$. 
ТеОРема 1.1. Имеют место следующие утверждения.

1. Пусть фиксировано число $0<p<2$. Тогда при $n \rightarrow \infty$ выполнено асимптотическое соотношение

$$
b_{n}(\beta, p)=\frac{(-1)^{n}}{n !}\left(\frac{n p}{e}\right)^{n p / 2} \beta^{n(2-p) / 2} \frac{e^{\beta / 2} \sqrt{2-p}}{\sqrt{2} \operatorname{sh}((\beta / 2) \sqrt{2-p})}(1+o(1)) .
$$

2. При $p=2$ и $n \rightarrow \infty$ имеет место асимптотическое соотношение

$$
b_{n}(\beta, 2)=\frac{(-1)^{n}}{n !}\left(\frac{2 n}{e}\right)^{n} \frac{\sqrt{2} e^{\beta / 2}}{\beta}(1+o(1)) .
$$

3. Пусть фиксировано число $2<p<p_{0}(\beta)$. Тогда при $n \rightarrow \infty$ справедливо асимптотическое соотношение

$$
b_{n}(\beta, p)=\frac{(-1)^{n}}{n !}\left(\frac{n p}{e}\right)^{n p / 2} \beta^{n(2-p) / 2} \frac{e^{\beta / 2} \sqrt{p-2}}{\sqrt{2} \sin ((\beta / 2) \sqrt{p-2})}(1+o(1)) .
$$

ЗАмЕчАниЕ 1. Возникновение порогового значения $p_{0}$ имеет то же объяснение, что и в работе [4]. А именно, можно показать, что при $p>p_{0}$ соответствующая экстремальная задача для функционала действия в методе Лапласа имеет однопараметрическое многообразие решений (ср. с леммой 10 в работе [4] и с теоремой 1 в статье [17]). Таким образом, в соответствии с п. 1.1 в рассматриваемой физической системе при $p=p_{0}=2+4 \pi^{2} / \beta^{2}$ происходит фазовый переход первого типа. Отсюда следует, что при $p=2 N$ для некоторых значений $\beta$ можно вычислить критическую размерность $N_{0}=p_{0} / 2=1+2 \pi^{2} / \beta^{2}$ (ср. с [1]). Отсюда также вытекает, что для фиксированного $p=2 N$ существует критическая температура $\beta_{0}=2 \pi / \sqrt{2 N-2}$, при которой в рассматриваемой физической системе происходит фазовый переход.

Исследованию точной асимптотики коэффициентов $b_{n}(\beta, p)$ в случае $p>p_{0}(\beta)$ при $n \rightarrow \infty$ целесообразно посвятить отдельную работу. Последняя задача имеет важное значение в $\phi^{2 N}$-теории поля при больших $p=2 N$.

ЗАмЕчАниЕ 2. Для порогового числа $p=p_{0}=2+4 \pi^{2} / \beta^{2}$ асимптотическое поведение коэффициентов $b_{n}(\beta, p)$ нужно исследовать отдельно. Здесь возникает так называемый вырожденный случай (см., в частности, теорему 2.3 в статье [18]). Логарифмическая асимптотика при этом имеет тот же вид.

ПРЕДЛОЖЕНИЕ 1.3. Справедливо соотношение

$$
\lim _{n \rightarrow \infty} \frac{1}{n} \ln \left(n ! n^{-n p_{0} / 2}(-1)^{n} b_{n}\left(\beta, p_{0}\right)\right)=\frac{p_{0}}{2} \ln \frac{p_{0}}{e}+\frac{2-p_{0}}{2} \ln \beta
$$

При $p=4$ мы получаем из п. 3 теоремы 1.1 следующее утверждение.

СлеДСтвиЕ 1.1. Пусть фиксировано число $0<\beta<\pi \sqrt{2}$. Тогда при $n \rightarrow \infty$ выполнено асимптотическое соотношение

$$
b_{n}(\beta, 4)=\frac{(-1)^{n}}{n !}\left(\frac{4 n}{e}\right)^{2 n} \beta^{-n} \frac{e^{\beta / 2}}{\sin (\beta / \sqrt{2})}(1+o(1)) .
$$


В теореме 18.3 из книги [13] была найдена следующая логарифмическая асимптотика при $p=4$ и $\beta>0$ :

$$
\lim _{n \rightarrow \infty} \frac{1}{n} \ln \left(n ! n^{-2 n}(-1)^{n} b_{n}(\beta, 4)\right)=-\alpha(\beta),
$$

при этом значение числа $\alpha(\beta)$ указано не было. Сравнивая формулы $(1.14)$ и (1.15), находим значение постоянной $\alpha(\beta)$ :

$$
\alpha(\beta)=\ln \frac{e^{2} \beta}{16} \quad \text { при } \quad 0<\beta<\pi \sqrt{2} .
$$

Отсюда следует, что $\alpha(\beta)$ - знакопеременная постоянная, а именно, $\alpha(\beta)>0$ при $16 / e^{2}<\beta<\pi \sqrt{2}, \alpha(\beta)=0$ при $\beta=16 / e^{2}$ и $\alpha(\beta)<0$ при $0<\beta<16 / e^{2}$. В силу предложения 1.3 равенство (1.16) выполнено и при $\beta=\pi \sqrt{2}$, т. е. $\alpha(\pi \sqrt{2})=\ln \left(e^{2} \pi \sqrt{2} / 16\right)$. Можно показать, что при $\beta>\pi \sqrt{2}$ формула (1.16) уже неверна.

\section{2. МЕТОД ЛАПЛАСА ДЛЯ ГАУССОВСКИХ МЕР В СЛУЧАЕ ГЛАДКИХ ФУНКЦИОНАЛОВ}

В настоящем разделе мы изложим два основных результата, при помощи которых доказываются теорема 1.1 и предложение 1.3. Необходимые сведения о гауссовских мерах в банаховых пространствах можно найти в книгах [41]-[44].

Пусть $(B,\|\cdot\|)$ - действительное сепарабельное банахово пространство, $\mathcal{B}$ - борелевская $\sigma$-алгебра в $B, B^{*}$ - сопряженное к $B$ пространство, $\langle\cdot, \cdot\rangle$ - линейная форма, устанавливающая двойственность между $B$ и $B^{*}$. Пусть $P_{A}$ - гауссовская мера на $\mathcal{B}$, имеющая нулевое среднее значение и инъективный ковариационный оператор $A: B^{*} \rightarrow B$. Напомним, что ковариационный оператор является симметричным, положительным и ядерным [42]. Инъективность оператора $A$ означает, что $\langle A y, y\rangle\rangle 0$ для всех $0 \neq y \in B^{*}$.

Обозначим через $S^{\prime}(x)$ и $S^{\prime \prime}(x)$ первую и вторую производные по Фреше числовой функции $S: B \rightarrow \mathbb{R}$ в точке $x$. Мы будем предполагать, что производные $S^{\prime}(x)$ и $S^{\prime \prime}(x)$ можно рассматривать как ограниченные линейные операторы из $B$ в $\mathbb{R}$ и из $B$ в $B^{*}$ соответственно, при этом будем употреблять запись

$$
S^{\prime}(x)[h]=\left\langle h, S^{\prime}(x)\right\rangle, \quad S^{\prime \prime}(x)[h, v]=\left\langle h, S^{\prime \prime}(x) v\right\rangle, \quad h, v \in B .
$$

Обозначим через $\operatorname{Dom}(S)$ область определения оператора $S$, а через $\operatorname{Ran}(S)$ - область значений оператора $S$, введем также $I$ - тождественный оператор в $B$. Пусть $\left(H_{A},\|\cdot\|_{A}\right)$ - гильбертово пространство, ассоциированное с ковариационным оператором $A$, т. е. пополнение области значений $\operatorname{Ran}(A)$ оператора $A$ относительно предгильбертовой нормы $\|y\|_{A}:=\sqrt{\langle A y, y\rangle}, y \in B^{*}[42]$.

\section{1. Точные асимптотики для гауссовских интегралов типа Лапласа.} Предположим, что выполнены следующие условия.

УсловиЕ 1. Пусть $f$ и $F$ - непрерывные действительные функции, заданные на $B$, такие, что для некоторых постоянных $c_{1}>0, c_{2} \geqslant 0,0 \leqslant c_{3}<(2\|A\|)^{-1}$ и $c_{4} \geqslant 0$ справедливы неравенства

$$
|f(x)| \leqslant c_{1} e^{c_{2}\|x\|^{2}}, \quad F(x) \geqslant-c_{3}\|x\|^{2}-c_{4}, \quad x \in B .
$$


УСловие 2. Функционал $\Lambda(x):=F(x)+\left\langle x, A^{-1} x\right\rangle / 2$ достигает своего минимума на $\operatorname{Dom}\left(A^{-1}\right)$ в конечном числе точек $x^{1}, x^{2}, \ldots, x^{m}$. Предположим, что

$$
\inf _{x \in H_{A}}\left\{F(x)+\frac{\|x\|_{A}^{2}}{2}\right\}
$$

также достигается только в точках $x^{1}, x^{2}, \ldots, x^{m}$.

УсловиЕ 3. В пространстве $B$ существуют окрестности $U_{i}$ точек $x^{i}$ такие, что функция $F$ трижды дифференцируема по Фреше в точках множеств $U_{i}$, причем $f\left(x^{i}\right) \neq 0$ для любого $i=1,2, \ldots, m$.

УсловиЕ 4. Для каждого $i=1,2, \ldots, m$ конечен и не равен нулю интеграл

$$
D_{i}:=\int_{B} e^{-\left\langle x, F^{\prime \prime}\left(x^{i}\right) x\right\rangle / 2} d P_{A}(x)
$$

Теорема 2.1. Пусть $P_{A}$ - гауссовская мера в В, имеющая нулевое среднее и инбективный ковариачионный оператор А. Пусть выполнены условия 1-4. Тогда $D_{i}>0$, для всех $i=1,2, \ldots, m$ имеют место равенства $x^{i}=-A F^{\prime}\left(x^{i}\right)$ и при $u \rightarrow \infty$ справедливо асимптотическое соотношение

$$
\int_{B} f(x) e^{-u^{2} F(x)} d P_{A}(u x)=e^{-u^{2} \Lambda\left(x^{1}\right)} \sum_{i=1}^{m} f\left(x^{i}\right) D_{i} \cdot(1+o(1)) .
$$

ЗАмЕЧАНИЕ 3. В условиях теоремы 2.1 часто бывает полезной формула

$$
D_{i}=\left[\operatorname{det}\left(I+A F^{\prime \prime}\left(x^{i}\right)\right)\right]^{-1 / 2}, \quad \operatorname{det}\left(I+A F^{\prime \prime}\left(x^{i}\right)\right):=\prod_{k=1}^{\infty}\left(1+\gamma_{i k}\right)
$$

где $\gamma_{i k}, k=1,2, \ldots,-$ собственные значения ядерного оператора $A F^{\prime \prime}\left(x^{i}\right): B \rightarrow B$, $i=1,2, \ldots, m[4],[8]$. Однако в настоящей работе нам будет удобнее вычислять постоянные $D_{i}$ непосредственно по формуле (2.1).

Теорема 2.1 представляет собой банаховозначный вариант известного результата Эллиса и Розен [18] об асимптотическом разложении гауссовского интеграла типа Лапласа в гильбертовом пространстве (см. также работу [3]).

\section{2. Логарифмические асимптотики для гауссовских мер и интегралов.} Принцип больших уклонений. Принцип больших уклонений для вероятностных мер и интегралов по ним (см. [3], [4], [45]-[48]) позволяет вычислить логарифмическую асимптотику интеграла из формулы (2.2) в тех случаях, когда вычисление точной асимптотики затруднено.

Теорема 2.2 (принцип больших уклонений для гауссовских мер). Пусть $P_{A}-$ гауссовская мера в банаховом пространстве $B$, имеющая нулевое среднее и инбективный ковариационный оператор $A$. Тогда семейство гауссовских мер

$$
\left\{P_{A}(u C), u>0\right\}, \quad C \in \mathcal{B},
$$


удовлетворяет принципу больших уклонений с функиионалом действия $I_{A}$, имеющим следующий вид:

$$
I_{A}(x)=\left\{\begin{array}{lll}
\|x\|_{A}^{2} / 2, & \text { если } & x \in H_{A}, \\
+\infty, & \text { если } & x \in B \backslash H_{A} .
\end{array}\right.
$$

Следовательно, для любой непрерывной функции $F: B \rightarrow \mathbb{R}$, удовлетворяющей условию 1, справедливо соотношение

$$
\lim _{u \rightarrow \infty} \frac{1}{u^{2}} \ln \int_{B} e^{-u^{2} F(x)} d P_{A}(u x)=-\inf _{x \in B}\left\{F(x)+I_{A}(x)\right\} .
$$

На основе соотношения (2.3) проводится вывод логарифмической асимптотики в формуле (1.13). Нам понадобится также следующий интересный результат, полезный при вычислениях, связанных с гауссовскими мерами.

ПреДЛОЖЕНИЕ 2.1. Пусть $P_{A}$ - гауссовская мера в сепарабельном банаховом пространстве $B$ с нулевым средним и инбективным ковариационным оператором $A$. Пусть $C: B \rightarrow B^{*}$ - непрерывный симметричный линейный оператор такой, что оператор $A^{-1}-C$ строго положителен, т.е. $\left\langle x,\left(A^{-1}-C\right) x\right\rangle>0$ для всех $x \neq 0$ из $\operatorname{Dom}\left(A^{-1}\right)$. Тогда справедливы следующие утверждения:

1) имеет место формула

$$
M:=\int_{B} e^{\langle x, C x\rangle / 2} P_{A}(d x)<\infty ;
$$

2) $M=[\operatorname{det}(I-A C)]^{-1 / 2}$ при условии, что детерминант конечен;

3) вероятностная мера

$$
\gamma(d x):=\frac{1}{M} e^{\langle x, C x\rangle / 2} P_{A}(d x)
$$

является гауссовской мерой на $B$ с нулевым средним и ингективным ковариационным оператором $A_{\gamma}:=\left(A^{-1}-C\right)^{-1} \equiv(I-A C)^{-1} A$.

ДокАЗАТЕЛЬСтво предложения проводится по схеме доказательства известных аналогичных утверждений. В случае гильбертова пространства данное предложение доказано в работе [18] (см. также [13]). В банаховом пространстве можно применить те же рассуждения, если использовать пространство $H_{A}$, одно из таких обобщений доказано в работе [49].

\section{3. ВЫБОР БАНАХОВА ФУНКЦИОНАЛЬНОГО ПРОСТРАНСТВА}

Доказательство теоремы 1.1 проводится при помощи теоремы 2.1. Покажем, что достаточно доказать теорему 1.1 в некотором более простом варианте, сформулированном для пространства $C[0,1]$.

3.1. Переход к пространству $C[0,1]$. Пусть $w_{0}(t):=\{w(t) \mid w(0)=0, w(1)=$ $0\}, t \in[0,1]$, обозначает стандартный броуновский мост [39]. Как известно, $w_{0}(t)-$ это гауссовский процесс с почти наверное непрерывными траекториями, нулевым средним и ковариационной функцией $\mathbf{E} w_{0}(t) w_{0}(s)=\min (t, s)-t s, t, s \in[0,1]$. Обозначим через $P_{w_{0}}$ распределение процесса $w_{0}(t)$ в пространстве $C[0,1]$. Легко видеть, что мера $P_{w_{0}}$ сосредоточена на подпространстве $C^{0}[0,1]:=\{x \in C[0,1]: x(0)=$ $x(1)=0\}$ пространства $C[0,1]$. Справедливо следующее утверждение. 
ЛЕмма 3.1. Пусть фиксированъ числа $p>0, \beta>0, n \geqslant 1$. Тогда выполнено следующее равенство:

$$
b_{n}(\beta, p)=\frac{(-1)^{n} e^{\beta / 2}}{n ! \sqrt{2 \pi}} \beta^{n(p+2) / 2} K(n, \beta, p),
$$

где

$$
\begin{aligned}
K(n, \beta, p)=\int_{-\infty}^{\infty} d v \int_{C[0,1]}\left(\int_{0}^{1}|x(s)+v|^{p} d s\right)^{n} \times \\
\\
\quad \times \exp \left\{-\frac{\beta^{2}}{2} \int_{0}^{1}(x(s)+v)^{2} d s\right\} P_{w_{0}}(d x) .
\end{aligned}
$$

ДокАЗАТЕЛьство. Используя формулу (1.8), получаем следующее равенство:

$$
b_{n}(\beta, p)=\frac{(-1)^{n} e^{\beta / 2}}{n ! \sqrt{2 \pi \beta}} K_{1}(n, \beta, p) \text {, }
$$

где

$$
\begin{aligned}
K_{1}(n, \beta, p)= & \int_{-\infty}^{\infty} d y \int_{C[0, \beta]}\left(\int_{0}^{\beta}|x(t)+y|^{p} d t\right)^{n} \times \\
& \times \exp \left\{-\frac{1}{2} \int_{0}^{\beta}(x(t)+y)^{2} d t\right\} P_{w}\{d x \mid x(0)=x(\beta)=0\} .
\end{aligned}
$$

В силу автомодельности винеровского процесса, выходящего из нуля [39], для любого фиксированного $\beta>0$ процессы $\{w(\beta s) \mid w(0)=0, w(\beta)=0\}, 0 \leqslant s \leqslant 1$, и $\left\{\beta^{1 / 2} w(s) \mid w(0)=0, w(1)=0\right\}, 0 \leqslant s \leqslant 1$, имеют одинаковые распределения. Учитывая этот факт, в результате замены переменной $t=\beta s$ в интеграле из формулы (3.4) получаем равенство

$$
\begin{aligned}
& K_{1}(n, \beta, p)=\beta^{n(p+2) / 2} \int_{-\infty}^{\infty} d y \int_{C[0,1]}\left(\int_{0}^{1}\left|x(s)+\frac{y}{\sqrt{\beta}}\right|^{p} d s\right)^{n} \times \\
& \times \exp \left\{-\frac{\beta^{2}}{2} \int_{0}^{1}\left(x(s)+\frac{y}{\sqrt{\beta}}\right)^{2} d s\right\} P_{w_{0}}(d x) .
\end{aligned}
$$

Сделаем замену переменной $v=y / \sqrt{\beta}$, получим $K_{1}(n, \beta, p)=\beta^{(n(p+2)+1) / 2} K(n, \beta, p)$, где $K(n, \beta, p)$ задано в (3.2). Отсюда и из равенства (3.3) вытекает формула (3.1). Лемма доказана.

3.2. Выбор банахова пространства и гауссовской меры. Цель настоящего пункта - преобразовать интеграл (3.2) так, чтобы к преобразованному интегралу была применима теорема 2.1.

Пусть $L^{p}=L^{p}[0,1]$ - множество измеримых функций, интегрируемых в $p$-й степени на отрезке $[0,1]$. Положим

$$
\|x\|_{p}:=\left(\int_{0}^{1}|x(t)|^{p} d t\right)^{1 / p}, \quad p>0 .
$$


Как известно, при $p \geqslant 1$ пространство $\left(L^{p},\|\cdot\|_{p}\right)$ является банаховым. Для фиксированного числа $p>0$ положим

$$
\widetilde{p}=\max (p, 2) \text {. }
$$

Таким образом, в наших рассуждениях $\widetilde{p} \geqslant 2$. Пусть $\widetilde{q}$ обозначает число, сопряженное к $\widetilde{p}$, т. е. $1 / \widetilde{p}+1 / \widetilde{q}=1$, очевидно, в нашем случае $2 \geqslant \widetilde{q}>1$. Напомним, что банахово пространство $L^{\widetilde{q}}$ является сопряженным к пространству $L^{\widetilde{p}}$. Как известно, каноническая билинейная форма $\langle\cdot, \cdot\rangle$ между пространствами $L^{\widetilde{p}}$ и $L^{\widetilde{q}}$ имеет интегральный вид:

$$
\langle h, g\rangle=\int_{0}^{1} h(t) g(t) d t, \quad h \in L^{\widetilde{p}}, \quad g \in L^{\widetilde{q}} .
$$

Мы будем рассматривать гауссовскую меру $P_{w_{0}}$, полагая ее заданной в пространстве $L^{\widetilde{p}}$, но имея в виду, что эта мера сосредоточена на $C[0,1]$.

Применяя теорему Фубини (см. также монографии [41] и [44]), несложно убедиться, что в нашем случае гауссовская мера $P_{w_{0}}$ имеет нулевое среднее и инъективный ковариационный оператор $A_{w_{0}}: L^{\widetilde{q}} \rightarrow L^{\widetilde{p}}$ интегрального типа:

$$
A_{w_{0}} x(t)=\int_{0}^{1}(\min (t, s)-t s) x(s) d s, \quad x \in L^{\widetilde{q}} .
$$

Отметим, что оператор $A_{w_{0}}$ зависит от $\widetilde{p}$, в частности $\operatorname{Dom}\left(A_{w_{0}}\right)=L^{\widetilde{q}}$, однако указывать эту зависимость мы не будем. Обозначим через $C^{2}[0,1]$ множество дважды дифференцируемых на отрезке $[0,1]$ функций. Утверждения следующих двух лемм хорошо известны [50], мы их приводим для удобства ссылок.

ЛЕмма 3.2. Область значений ковариационного оператора $A_{w_{0}}$, определенного в формуле (3.7), имеет вид

$$
\operatorname{Ran}\left(A_{w_{0}}\right)=\left\{x \in C^{2}[0,1]: x(0)=x(1)=0\right\} .
$$

Обратнъй оператор задается формулой

$$
\left[A_{w_{0}}^{-1} y\right](t)=-y^{\prime \prime}(t), \quad y \in \operatorname{Ran}\left(A_{w_{0}}\right), \quad t \in[0,1] .
$$

ЛЕмма 3.3. Собственные числа $\left\{\lambda_{k}\right\}_{k=1}^{\infty}$ ковариационного оператора $A_{w_{0}}$ имеют следующий вид:

$$
\lambda_{k}=\frac{1}{\pi^{2} k^{2}}, \quad k=1,2, \ldots,
$$

им соответствуют собственные функиии

$$
\phi_{k}(t)=\sqrt{2} \sin (\pi k t), \quad k=1,2, \ldots,
$$

которые составляют ортонормированный базис в $L^{2}$.

Возьмем в качестве банахова пространства $B$ из теоремы 2.1 прямое произведение

$$
B=\mathbb{R} \times L^{\widetilde{p}}
$$


двух банаховых пространств - одномерного пространства $(\mathbb{R},|\cdot|)$ и пространства $\left(L^{\widetilde{p}},\|\cdot\|_{\widetilde{p}}\right)[51]$. Элемент $z \in B$ будем записывать в следующем виде:

$$
z=(y, x), \quad y \in \mathbb{R}, \quad x \in L^{\widetilde{p}}
$$

Напомним, что $B$ является банаховым пространством относительно нормы $\|\cdot\|_{B}$, заданной равенством

$$
\|z\|_{B}^{\widetilde{p}}:=|y|^{\widetilde{p}}+\|x\|_{\widetilde{p}}^{\widetilde{p}}, \quad z=(y, x) \in B .
$$

В качестве гауссовской меры $P_{A}(d z)$ на пространстве (3.12) возьмем гауссовскую меру-произведение

$$
P_{A}(d z)=\nu_{\beta}(d z) \equiv \nu_{\beta}(d y, d x):=\mu_{\beta}(d y) \times P_{w_{0}}(d x), \quad z=(y, x),
$$

где

$$
\mu_{\beta}(d y)=\frac{\beta}{\sqrt{2 \pi}} e^{-\beta^{2} y^{2} / 2} d y, \quad y \in \mathbb{R}
$$

- одномерная гауссовская мера на прямой с нулевым средним и дисперсией $1 / \beta^{2}$, a $P_{w_{0}}$ - определенная выше гауссовская мера на $L^{\widetilde{p}}$. Меры-произведения рассмотрены в книгах [42], [51].

Сопряженным к пространству (3.12) является пространство $B^{*}=\mathbb{R} \times L^{\widetilde{q}}$. Каноническую билинейную форму между пространствами $B$ и $B^{*}$ обозначим через $\langle\cdot, \cdot\rangle_{B}$. Несложно вывести, используя (3.14), что в гильбертовом случае при $\widetilde{p}=2$ (и, следовательно, $\widetilde{q}=2$ ) выполнено равенство

$$
\left\langle z_{1}, z_{2}\right\rangle_{B}=y_{1} y_{2}+\left\langle x_{1}, x_{2}\right\rangle, \quad z_{1}=\left(y_{1}, x_{1}\right) \in B, \quad z_{2}=\left(y_{2}, x_{2}\right) \in B^{*},
$$

где билинейная форма $\langle\cdot, \cdot\rangle$ определена в (3.6).

ЛЕмма 3.4. Имеют место следуюшие утверждения.

1. Мера $\nu_{\beta}(d y, d x)$, заданная в $(3.15)$, является гауссовской мерой с нулевым средним и ковариационным оператором $A_{\nu}: B^{*} \rightarrow B$ следующего вида:

$$
A_{\nu}(y, x)=\left(\frac{y}{\beta^{2}}, A_{w_{0}} x\right), \quad(y, x) \in B^{*},
$$

где оператор $A_{w_{0}}$ задан в формуле (3.7).

2. Область значений ковариачионного оператора $A_{\nu}$ есть

$$
\operatorname{Ran}\left(A_{\nu}\right)=\mathbb{R} \times \operatorname{Ran}\left(A_{w_{0}}\right),
$$

где $\operatorname{Ran}\left(A_{w_{0}}\right)$ определена в (3.8). Обратный оператор задается формулой

$$
A_{\nu}^{-1}(y, x)=\left(\beta^{2} y, A_{w_{0}}^{-1} x\right)=\left(\beta^{2} y,-x^{\prime \prime}\right), \quad(y, x) \in \operatorname{Ran}\left(A_{\nu}\right) .
$$

ДокАзАтЕльство. Утверждение 1 получаем, используя формулы (3.15)-(3.17) и вид характеристического функционала гауссовской меры (см. предложение 2.8 в книге [42]). Утверждение 2 вытекает непосредственно из первого утверждения и формул (3.8), (3.9). 
3.3. Выбор основного функционала. Учитывая формулу (3.2), возьмем в качестве функционала $F$ из теоремы 2.1 следующий функционал:

$$
F(z) \equiv F(y, x):=\frac{\beta^{2}}{2} \int_{0}^{1}(x(t)+y)^{2} d t-\ln \int_{0}^{1}|x(t)+y|^{p} d t-\frac{\beta^{2} y^{2}}{2},
$$

где $z=(y, x) \in B$. Приводя подобные члены, заключаем, что

$$
F(y, x)=\frac{\beta^{2}}{2}\left(\int_{0}^{1} x^{2}(t) d t+2 y \int_{0}^{1} x(t) d t\right)-\ln \int_{0}^{1}|x(t)+y|^{p} d t .
$$

ЛЕмма 3.5. Для фиксированных чисел $p>0, \beta>0, n \geqslant 1$ выполнено равенство

$$
K(n, \beta, p)=n^{n p / 2} \frac{\sqrt{2 \pi}}{\beta} K_{2}(n, \beta, p),
$$

где

$$
K_{2}(n, \beta, p)=\int_{B} e^{-n F(z)} d \nu_{\beta}(z \sqrt{n}) .
$$

ДокАзАТЕЛЬСтво. Положив в формуле (3.2) y вместо $v$ и использовав соотношения (3.15), (3.16) и (3.22), получаем цепочку равенств

$$
\begin{aligned}
K(n, \beta, p)= & n^{n p / 2} \frac{\sqrt{2 \pi}}{\beta} \int_{-\infty}^{\infty} \mu_{\beta}(d y) \int_{L^{\tilde{p}}} \exp \left\{-\frac{\beta^{2}}{2}\left(\int_{0}^{1} x^{2}(t) d t+2 y \int_{0}^{1} x(t) d t\right)+\right. \\
& \left.+n \ln \int_{0}^{1}\left|\frac{x(t)+y}{\sqrt{n}}\right|^{p} d t\right\} P_{w_{0}}(d x)= \\
= & n^{n p / 2} \frac{\sqrt{2 \pi}}{\beta} \int_{\mathbb{R} \times L^{\tilde{p}}} \exp \left\{-n F\left(\frac{y}{\sqrt{n}}, \frac{x}{\sqrt{n}}\right)\right\} \nu_{\beta}(d y, d x) .
\end{aligned}
$$

Из последнего равенства и формул (3.12), (3.13) следует утверждение леммы.

Нам понадобится следующее полезное неравенство для функционала $\|\cdot\|_{p}$. Напомним, что $\|x\|_{\infty}=\sup _{t \in[0,1]}|x(t)|$ для $x \in C[0,1]$.

ЛЕмма 3.6. Пусть фиксировань числа $0<s<r \leqslant \infty$. Если $\|x\|_{r}<\infty$, то справедливо неравенство

$$
\|x\|_{s} \leqslant\|x\|_{r} .
$$

ДокАЗАТЕЛЬство леммы при $r<\infty$ имеется в книге [52]. При $r=\infty$ неравенство (3.25) очевидно.

\section{4. НАЧАЛО ДОКАЗАТЕЛЬСТВА ТЕОРЕМЫ 1.1. РЕШЕНИЕ ЭКСТРЕМАЛЬНОЙ ЗАДАЧИ}

Мы применим теорему 2.1 к модельному интегралу Лапласа (3.24), полагая в нем $B=\mathbb{R} \times L^{\widetilde{p}}, P_{A}(d z)=\nu_{\beta}(d z), f(x) \equiv 1$, при большом параметре $u=\sqrt{n}$. Доказательство всех трех пунктов теоремы 1.1 до определенного момента проводится единым образом, при этом некоторые утверждения ниже мы формулируем и доказываем сразу для общего случая $0<p<\infty$. Очевидно, что функционал (3.21) и ковариационный оператор (3.18) удовлетворяют условию 1. 
Далее мы используем определение производной по Фреше, данное в монографии [53], где допускается, чтобы рассматриваемый оператор был определен всего лишь на некотором всюду плотном подмножестве банахова пространства, при этом возможны значения производных, равные $\pm \infty$ (см. определение 1 в [54]). Функционал $F(y, x)$ согласно (3.21) представляет собой линейную комбинацию квадратичного функционала и логарифма интегрального функционала. Как известно, интегральные функционалы дифференцируемы по Фреше [52]-[54].

Обозначим через $\mathcal{X}$ множество функций из $C[0,1]$, имеющих конечное число нулей. Очевидно, множество $\mathcal{X}$ всюду плотно в $C[0,1]$ и, следовательно, в $L^{\widetilde{p}}$. Положим $\mathcal{Z}=\mathbb{R} \times \mathcal{X}$.

Лемма 4.1. Для фиксированных чисел $\beta>0$ и $p>0$ функиионал $F(z)$, заданный в (3.21), трижды дифферениируем по Фреше в каждой точке $z \in \mathcal{Z}$. При $p \geqslant 2$ u $z \in \mathcal{Z}$ оператор $F^{\prime \prime}(z): B \rightarrow B^{*}$ является ограниченным.

ДокАЗАТЕЛЬство леммы мы получаем, применяя теорему о суперпозиции дифференцируемых отображений [54] и известные формулы для первых двух производных функционала $\widehat{F}(x)=\int_{0}^{1}|x(t)|^{p} d t$.

Решение экстремальной задачи. Согласно формулам (3.17)-(3.20) основная экстремальная задача из условия 2 имеет вид

$$
\Lambda(z):=F(z)+\frac{1}{2}\left\langle z, A_{\nu}^{-1} z\right\rangle_{B} \equiv F(y, x)+\frac{\beta^{2} y^{2}}{2}+\frac{1}{2}\left\langle x, A_{w_{0}}^{-1} x\right\rangle \rightarrow \inf _{z},
$$

где $z=(y, x) \in \operatorname{Ran}\left(A_{\nu}\right)$. Учитывая (3.21), заключаем, что эту задачу можно записать в следующей форме:

$$
\Lambda(y, x) \equiv \frac{\beta^{2}}{2} \int_{0}^{1}(x(t)+y)^{2} d t-\ln \int_{0}^{1}|x(t)+y|^{p} d t+\frac{1}{2}\left\langle x, A_{w_{0}}^{-1} x\right\rangle \rightarrow \inf _{(x, y)},
$$

где $(y, x) \in \mathbb{R} \times \operatorname{Ran}\left(A_{w_{0}}\right)$.

Лемма 4.2. Пусть фиксированъ числа $\beta>0 u 0<p \leqslant p_{0}(\beta)$. Тогда минимум в экстремальной задаче (4.2) достигается только в двух точках

$$
z_{0}=\left(y_{0}, 0\right)=\left(\frac{\sqrt{p}}{\beta}, 0\right), \quad-z_{0}=-\left(\frac{\sqrt{p}}{\beta}, 0\right) .
$$

При этом

$$
\min _{(y, x) \in \mathbb{R} \times \operatorname{Ran}\left(A_{w_{0}}\right)} \Lambda(y, x)=\Lambda\left(z_{0}\right)=\frac{p}{2}-\frac{p}{2} \ln \frac{p}{\beta^{2}} .
$$

Кроме того,

$$
\inf _{z \in H_{A_{\nu}}}\left\{F(z)+\frac{\|z\|_{A_{\nu}}^{2}}{2}\right\}
$$

также достигается только в точках $\pm z_{0}$.

ДокАЗАТЕЛЬСТво леммы довольно сложное, мы его приведем после того, как докажем несколько вспомогательных утверждений.

Для $1 \leqslant p \leqslant \infty$ обозначим через $\left\|A_{w_{0}}\right\|_{p}$ норму ковариационного оператора $A_{w_{0}}:\left(L^{p}\right)^{*} \rightarrow L^{p}$. 
ЛЕмма 4.3. Пусть фиксированы числа $\beta>0$ и р >0. Тогда справедливы следующие утверждения.

1. Минимум в задаче (4.2) достигается.

2. Выполнено неравенство (оценка сверху)

$$
\min _{(y, x) \in \mathbb{R} \times \operatorname{Ran}\left(A_{w_{0}}\right)} \Lambda(y, x) \leqslant \frac{p}{2}-\frac{p}{2} \ln \frac{p}{\beta^{2}}
$$

причем знак равенства имеет место тогда и только тогда, когда $(y, x)= \pm\left(y_{0}, 0\right)$.

ДокАзАтЕльство. Используя доказательство теоремы Ландау-Маркуса-Шеппа-Ферника из статьи [55], убеждаемся, что справедливо неравенство

$$
\left\langle x, A_{w_{0}}^{-1} x\right\rangle \geqslant \frac{\|x\|_{\widetilde{p}}^{2}}{\left\|A_{w_{0}}\right\|_{\tilde{p}}}, \quad x \in L^{\widetilde{p}},
$$

где квадратичную форму $\left\langle x, A_{w_{0}}^{-1} x\right\rangle$ мы распространили по непрерывности на все пространство $L^{\widetilde{p}}$. Из формул $(3.5)$, (4.6) и первой теоремы Вейерштрасса (см. замечание 9.1 в [53]) следует, что минимум в задаче (4.2) достигается, поскольку в силу (3.25) мы имеем $\|x\|_{2} \geqslant\|x\|_{p}$ при $0<p \leqslant 2$. Первое утверждение леммы доказано.

Завершим доказательство леммы. Учитывая неравенство $\left\langle x, A_{w_{0}}^{-1} x\right\rangle \geqslant 0$, заключаем, что справедлива следующая оценка сверху:

$$
\min _{(y, x) \in \mathbb{R} \times \operatorname{Ran}\left(A_{w_{0}}\right)} \Lambda(y, x) \leqslant \min _{y \in \mathbb{R}} \Lambda(y, 0) .
$$

Нетрудно видеть, что функция $\Lambda(y, 0)=\beta^{2} y^{2} / 2-p \ln |y|$ достигает своего минимума при $y \in \mathbb{R}$ в двух точках $\pm y_{0}= \pm \sqrt{p} / \beta$, при этом

$$
\Lambda\left(y_{0}, 0\right)=\Lambda\left(-y_{0}, 0\right)=\frac{p}{2}-\frac{p}{2} \ln \frac{p}{\beta^{2}} .
$$

Отсюда и из неравенства (4.7) вытекает оценка (4.5). Знак равенства в (4.5) имеет место тогда и только тогда, когда $(y, x)= \pm\left(y_{0}, 0\right)$, так как $\left\langle x, A_{w_{0}}^{-1} x\right\rangle>0$ при $0 \neq x \in \operatorname{Ran}\left(A_{w_{0}}\right)$. Лемма доказана.

Используя методы вариационного исчисления [54] и учитывая лемму 4.1 и п. 2 леммы 4.3, исследуем гладкую экстремальную задачу (4.2). Опишем стационарные точки этой задачи. Частные производные и производная по Фреше функции двух переменных определены в книге [54], напомним также, что $\mathcal{Z}=\mathbb{R} \times \mathcal{X}$.

Лемма 4.4. Пусть фиксированы числа $\beta>0$ и р>0. Тогда справедливы следующие утверждения.

1. Функиия $\Lambda(y, x)$, заданная в (4.2), дваждъ дифберениируема по Фреше в каждой точке $z=(y, x) \in \mathcal{Z}$. Первая производная имеет вид

$$
\Lambda^{\prime}(y, x)[g, h]=\frac{\partial}{\partial y} \Lambda(y, x) g+\frac{\partial}{\partial x} \Lambda(y, x)[h], \quad g \in \mathbb{R}, \quad h \in \operatorname{Ran}\left(A_{w_{0}}\right)
$$


¿əe

$$
\begin{aligned}
& \frac{\partial}{\partial y} \Lambda(y, x)=\beta^{2} \int_{0}^{1} x(t) d t+\beta^{2} y-\frac{p \int_{0}^{1}|x(t)+y|^{p-1} \operatorname{sgn}(x(t)+y) d t}{\int_{0}^{1}|x(t)+y|^{p} d t} \\
& \frac{\partial}{\partial x} \Lambda(y, x)=\beta^{2} x(t)+\beta^{2} y+A_{w_{0}}^{-1} x-\frac{p|x(t)+y|^{p-1} \operatorname{sgn}(x(t)+y)}{\int_{0}^{1}|x(t)+y|^{p} d t} .
\end{aligned}
$$

2. Множеству стационарных точек, т.е. множеству решений системы уравнений

$$
\frac{\partial}{\partial y} \Lambda(y, x)=0, \quad \frac{\partial}{\partial x} \Lambda(y, x)=0,
$$

принадлежат точки $\pm z_{0}= \pm\left(y_{0}, 0\right)$, заданные в $(4.3)$.

3. Квадратичная форма, построенная по производной $\Lambda^{\prime \prime}\left(z_{0}\right)$, имеет вид

$$
\left\langle z, \Lambda^{\prime \prime}\left(z_{0}\right) z\right\rangle_{B}=\beta^{2}(p-2)\left(\langle x, \mathbf{1}\rangle^{2}-\langle x, x\rangle\right)+\left\langle x, A_{w_{0}}^{-1} x\right\rangle+2 \beta^{2}(y+\langle x, \mathbf{1}\rangle)^{2},
$$

где $z=(y, x)$ и $\mathbf{1}$ - функция, тождественно равная единице на отрезке [0, 1].

ДокАзАтЕльство. Все три утверждения леммы выводятся в результате несложных вычислений при помощи теоремы о суперпозиции дифференцируемых отображений [54] и известных формул для первых двух производных функционала $\widehat{F}(x)=$ $\int_{0}^{1}|x(t)|^{p} d t$. Отметим, что стационарные точки $\pm z_{0}$ в п. 2 были найдены как решения системы (4.9), являющиеся постоянными функциями. При этом учитывалось, что единственная постоянная функция, принадлежащая множеству $\operatorname{Ran}\left(A_{w_{0}}\right),-$ это функция, тождественно равная нулю на отрезке [0,1]. Лемма доказана.

Определим оператор $\Lambda_{0} \equiv \Lambda_{0}\left(z_{0}\right): L^{2} \rightarrow L^{2}$ следующим образом:

$$
\begin{aligned}
\Lambda_{0} x & =\beta^{2}(p-2)(\langle x, \mathbf{1}\rangle-x)+A_{w_{0}}^{-1} x \equiv \\
& \equiv-x^{\prime \prime}(t)-\beta^{2}(p-2) x(t)+\beta^{2}(p-2)\langle x, \mathbf{1}\rangle, \quad x \in \operatorname{Ran}\left(A_{w_{0}}\right) .
\end{aligned}
$$

Тогда формулу (4.10) можно записать как

$$
\left\langle z, \Lambda^{\prime \prime}\left(z_{0}\right) z\right\rangle_{B}=\left\langle x, \Lambda_{0} x\right\rangle+2 \beta^{2}(y+\langle x, \mathbf{1}\rangle)^{2}, \quad z=(y, x) .
$$

Некоторые полезные свойства оператора (4.11) описаны в следующей лемме.

ЛЕмма 4.5. Справедливы следующие утверждения.

1. Для фиксированных чисел $\beta>0 u 0<p<\infty$ квадратичная форма $\left\langle x, \Lambda_{0} x\right\rangle$ ограничена снизу. А именно, при $0<p \leqslant 2$ имеет место ощенка

$$
\left\langle x, \Lambda_{0} x\right\rangle \geqslant \pi^{2}\langle x, x\rangle \geqslant 0, \quad x \in \operatorname{Ran}\left(A_{w_{0}}\right) .
$$

При $2 \leqslant p<\infty$ выполнено неравенство

$$
\left\langle x, \Lambda_{0} x\right\rangle \geqslant\left(\pi^{2}+\beta^{2}(2-p)\right)\langle x, x\rangle, \quad x \in \operatorname{Ran}\left(A_{w_{0}}\right) .
$$

2. Пусть биксированы числа $\beta>0$ и $2<p<\infty$. Тогда оператор $\Lambda_{0}$ является симметричным и имеет чисто дискретный простой спектр. Множество собственных чисел оператора $\Lambda_{0}$ есть $\left\{\gamma_{k}\right\}_{k=1}^{\infty} \cup\left\{\widetilde{\gamma}_{k}\right\}_{k=1}^{\infty}$, где

$$
\gamma_{k}=4 k^{2} \pi^{2}+\beta^{2}(2-p), \quad k=1,2, \ldots,
$$


им соответствуют нормированные в $L^{2}$ собственные функции

$$
e_{k}(t)=\sqrt{2} \sin (2 \pi k t), \quad k=1,2, \ldots ;
$$

собственные числа $\widetilde{\gamma}_{k}$ имеют вид

$$
\widetilde{\gamma}_{k}=4 \theta_{k}^{2}+\beta^{2}(2-p), \quad k=1,2, \ldots ;
$$

здесъ $0<\theta_{1}<\theta_{2}<\cdots<\theta_{k}<\cdots$ - однократные положительнье корни трансцендентного уравнения

$$
\operatorname{tg} \theta=\theta+\frac{4 \theta^{3}}{\beta^{2}(2-p)}, \quad \theta>0 .
$$

Оператор $\Lambda_{0}$ имеет нулевое ядро при $p \neq 2+4 \pi^{2} k^{2} / \beta^{2}, k=1,2, \ldots$.

3. Для фиксированных чисел $\beta>0$ и $0<p<p_{0}(\beta)$, где $p_{0}(\beta)$ задано в (1.9), имеют место неравенства

$$
\begin{gathered}
0<\theta_{1}<\pi, \quad 4 \theta_{1}^{2}+\beta^{2}(2-p)>0 \\
\left\langle x, \Lambda_{0} x\right\rangle \geqslant\left(4 \theta_{1}^{2}+\beta^{2}(2-p)\right)\langle x, x\rangle, \quad x \in \operatorname{Ran}\left(A_{w_{0}}\right) .
\end{gathered}
$$

При $p=p_{0}(\beta)$ выполнены соотношения

$$
\theta_{1}=\pi, \quad 4 \theta_{1}^{2}+\beta^{2}\left(2-p_{0}\right)=0, \quad\left\langle x, \Lambda_{0} x\right\rangle \geqslant 0, \quad x \in \operatorname{Ran}\left(A_{w_{0}}\right) .
$$

ДоказАтельство. В силу неравенства Шварца $\langle x, \mathbf{1}\rangle^{2} \leqslant\langle x, x\rangle$ для всех $x \in L^{2}$. Отсюда и из (4.11) вытекает следующее неравенство при $0<p \leqslant 2$ :

$$
\left\langle x, \Lambda_{0} x\right\rangle \geqslant\left\langle x, A_{w_{0}}^{-1} x\right\rangle, \quad x \in \operatorname{Ran}\left(A_{w_{0}}\right) .
$$

Согласно лемме 3.2 оператор $A_{w_{0}}^{-1}$ является симметричным инъективным оператором Штурма-Лиувилля в пространстве $L^{2}$ и имеет чисто дискретный простой спектр [56]. В силу леммы 3.3 собственные числа оператора $A_{w_{0}}^{-1}$ имеют вид $1 / \lambda_{k}=$ $\pi^{2} k^{2}, k=1,2, \ldots$, а соответствующие собственные векторы (3.11) составляют ортонормированный базис в $L^{2}$. Следовательно, выполнено неравенство

$$
\left\langle x, A_{w_{0}}^{-1} x\right\rangle \geqslant \pi^{2}\langle x, x\rangle, \quad x \in \operatorname{Ran}\left(A_{w_{0}}\right) .
$$

Из формул (4.22) и (4.23) вытекает оценка (4.13).

Докажем неравенство (4.14). При $2 \leqslant p<\infty$ в силу формул (4.11) и (4.23) выполнены неравенства

$$
\left\langle x, \Lambda_{0} x\right\rangle \geqslant\left\langle x, A_{w_{0}}^{-1} x\right\rangle-\beta^{2}(p-2)\langle x, x\rangle \geqslant\left(\pi^{2}+\beta^{2}(2-p)\right)\langle x, x\rangle, \quad x \in \operatorname{Ran}\left(A_{w_{0}}\right) .
$$

Неравенство (4.14), а вместе с ним и утверждение 1 леммы доказаны.

Докажем утверждение 2. Оператор (4.11) представляет собой сумму симметричного оператора Штурма-Лиувилля $-d^{2} / d t^{2}-\beta^{2}(p-2)[56]$ и одномерного оператора $\langle\cdot, \mathbf{1}\rangle \mathbf{1}$ с областью значений $\{k \mathbf{1}: k \in \mathbb{R}\} \subset L^{2}$. Следовательно, согласно общей теории оператор $\Lambda_{0}$ является симметричным и имеет чисто дискретный простой спектр.

Рассмотрим для $p>2$ задачу $\Lambda_{0} x=\gamma x$ на собственные числа оператора $\Lambda_{0}$, т. е. задачу

$$
\beta^{2}(p-2)(\langle x, \mathbf{1}\rangle-x)+A_{w_{0}}^{-1} x=\gamma x, \quad x \in \operatorname{Ran}\left(A_{w_{0}}\right) .
$$


В силу леммы 3.2 эту задачу можно записать в виде следующей граничной задачи:

$$
\begin{gathered}
x^{\prime \prime}(t)+\mu x(t)-\beta^{2}(p-2) \int_{0}^{1} x(s) d s=0, \quad t \in[0,1], \\
x(0)=x(1)=0,
\end{gathered}
$$

где

$$
\mu:=\beta^{2}(p-2)+\gamma
$$

Рассмотрим далее отдельно два случая.

Случай 1. Пусть функция $x \in \operatorname{Ran}\left(A_{w_{0}}\right)$ такова, что

$$
\int_{0}^{1} x(t) d t=0
$$

Тогда задача (4.24) принимает следующий вид:

$$
\begin{aligned}
x^{\prime \prime}(t)+\mu x(t) & =0, \quad t \in[0,1], \\
x(0)=x(1) & =0 .
\end{aligned}
$$

Легко видеть, что (4.27) представляет собой задачу на собственные числа для оператора $A_{w_{0}}^{-1}$. Используя формулы $(3.10),(3.11)$, убеждаемся, что задача $(4.26),(4.27)$ имеет решения $\left(\mu_{k}, e_{k}\right)$, где

$$
\mu_{k}=\frac{1}{\lambda_{2 k}}=4 k^{2} \pi^{2}, \quad e_{k}(t)=\phi_{2 k}(t)=\sqrt{2} \sin (2 \pi k t), \quad k=1,2, \ldots,
$$

так как $\int_{0}^{1} \phi_{2 k}(t) d t=0, \int_{0}^{1} \phi_{2 k-1}(t) d t \neq 0$ для всех $k=1,2, \ldots$. Таким образом, в силу формул (4.25) и (4.28) мы получаем в рассматриваемом случае собственные числа (4.15) и нормированные в $L^{2}$ собственные функции (4.16).

Случай 2. Пусть функция $x \in \operatorname{Ran}\left(A_{w_{0}}\right)$ такова, что

$$
c:=\int_{0}^{1} x(t) d t \neq 0
$$

Тогда задачу (4.24) можно записать в следующем виде:

$$
\begin{gathered}
x^{\prime \prime}(t)+\mu x(t)-\beta^{2}(p-2) c=0, \quad t \in[0,1], \\
x(0)=x(1)=0 .
\end{gathered}
$$

В результате замены переменной

$$
g(t)=x(t)-\frac{\beta^{2}(p-2) c}{\mu}, \quad t \in[0,1],
$$

задача (4.30) принимает вид

$$
\begin{gathered}
g^{\prime \prime}(t)+\mu g(t)=0, \quad t \in[0,1], \\
g(0)=g(1)=\frac{\beta^{2}(2-p) c}{\mu} .
\end{gathered}
$$


Учитывая результаты из книги [56], часть 3 , гл. 2, рассмотрим далее отдельно случаи $\mu>0$ и $\mu \leqslant 0$.

Пусть спектральный параметр $\mu$ из (4.25) положителен, положим

$$
\psi^{2}:=\mu=\beta^{2}(p-2)+\gamma>0, \quad \psi>0 .
$$

Решая задачу (4.32) и учитывая замену (4.31), убеждаемся, что единственное решение задачи (4.30) имеет вид

$$
x(t)=\frac{\beta^{2}(2-p) c}{\psi^{2}}\left(\cos (\psi t)+\frac{1-\cos \psi}{\sin \psi} \sin (\psi t)-1\right), \quad t \in[0,1] .
$$

Используем формулы (4.29) и (4.34), получим уравнение для параметра $\psi>0$ :

$$
\operatorname{tg} \frac{\psi}{2}=\frac{\psi}{2}+\frac{\psi^{3}}{2 \beta^{2}(2-p)} .
$$

Полагая $\theta=\psi / 2$, заключаем, что данное уравнение принимает вид (4.18). Графически можно показать, что уравнение (4.18) имеет однократные положительные корни $0<\theta_{1}<\theta_{2}<\cdots<\theta_{k}<\cdots$. Отсюда, используя (4.33), получаем собственные числа $\widetilde{\gamma}_{k}$, записанные в формуле (4.17).

Несложно доказать, что если $\mu \leqslant 0$, то задача (4.30) при условии (4.29) не имеет решений для $p>2$.

Таким образом, при условии (4.29) задача (4.30) имеет в качестве собственных только числа $\mu_{k}=4 \theta_{k}^{2}=\beta^{2}(p-2)+\widetilde{\gamma}_{k}, k=1,2, \ldots$. На основе полученных выше результатов мы выводим основное утверждение п. 2 леммы о собственных числах. Утверждение о ядре легко доказать, используя формулу (4.15) и рассуждения в случае $\mu>0$ при $\gamma=0$.

Докажем утверждение 3. Пусть фиксировано число $p<2+4 \pi^{2} / \beta^{2}$. Рассмотрим кубическую кривую

$$
f(\theta):=\theta+\frac{4 \theta^{3}}{\beta^{2}(2-p)}, \quad \theta \in \mathbb{R},
$$

из правой части уравнения (4.18). Легко видеть, что уравнение $f(\theta)=0$ имеет три действительных корня

$$
\theta_{0}=0, \quad \pm \theta_{*}= \pm \frac{\beta \sqrt{p-2}}{2},
$$

из которых только один (корень $\theta_{*}$ ) строго положителен. Поскольку $p<p_{0}(\beta)$, справедлива оценка $0<\theta_{*}<\pi$. Учитывая данную оценку, применяя неравенство $\operatorname{tg} \theta<\theta$ при $0<\theta<\pi / 2$ и решая графически уравнение (4.18), заключаем, что имеют место неравенства $\pi / 2<\theta_{*}<\theta_{1}<\pi$. Тем самым мы доказали первое неравенство в формуле (4.19). Второе неравенство в (4.19) вытекает из первого и оценки $p<p_{0}(\beta)$.

Из п. 2 доказываемой леммы и неравенств (4.19) следует, что минимальное собственное число квадратичной формы $\left\langle x, \Lambda_{0} x\right\rangle$ равно $\widetilde{\gamma}_{1}=4 \theta_{1}^{2}+\beta^{2}(2-p)>0$. Отсюда в силу свойств оператора $\Lambda_{0}$ вытекает неравенство (4.20). Соотношения (4.21) доказываются аналогично соотношениям (4.19), (4.20). Третье утверждение, а вместе с ним и лемма доказаны полностью. 
Лемма 4.6. Пусть биксированъ числа $\beta>0 u 0<p \leqslant p_{0}(\beta)$. Тогда оператор $\Lambda^{\prime \prime}\left(z_{0}\right)$, заданный в $(4.10)$, является строго положительным при $0<p<p_{0}(\beta)$ и положительным при $p=p_{0}(\beta)$.

ДокАзАТЕЛЬство. Утверждение леммы следует непосредственно из формулы (4.12) и п. 1, 2 леммы 4.5.

Введем функцию

$$
\Lambda_{1}(y, x):=\frac{\beta^{2}}{2} \int_{0}^{1}(x(t)+y)^{2} d t-\ln \int_{0}^{1}|x(t)+y|^{p} d t, \quad(y, x) \in \mathbb{R} \times \operatorname{Ran}\left(A_{w_{0}}\right) .
$$

Легко видеть, что в силу (4.2) выполнено равенство

$$
\Lambda_{1}(y, x)=\Lambda(y, x)-\frac{1}{2}\left\langle x, A_{w_{0}}^{-1} x\right\rangle, \quad(y, x) \in \mathbb{R} \times \operatorname{Ran}\left(A_{w_{0}}\right) .
$$

Лемма 4.7. Пусть фиксированъ числа $\beta>0$ u $0<p \leqslant 2$. Тогда имеет место следующая оченка снизу:

$$
\min _{(y, x) \in \mathbb{R} \times \operatorname{Ran}\left(A_{w_{0}}\right)} \Lambda(y, x) \leqslant \min _{(y, x) \in \mathbb{R} \times \operatorname{Ran}\left(A_{w_{0}}\right)} \Lambda_{1}(y, x)=\Lambda_{1}\left(y_{0}, 0\right)=\frac{p}{2}-\frac{p}{2} \ln \frac{p}{\beta^{2}} .
$$

Минимум в последней экстремальной задаче в (4.37) достигается только в двух mочках $(y, x)= \pm\left(y_{0}, 0\right)$, где $y_{0}=\sqrt{p} / \beta$.

ДоказАТЕЛьство. В силу формул (4.2) и (4.35) мы имеем $\Lambda(y, x) \leqslant \Lambda_{1}(y, x)$ для всех $(y, x) \in \mathbb{R} \times \operatorname{Ran}\left(A_{w_{0}}\right)$. Отсюда, очевидно, вытекает первое неравенство в формуле (4.37).

Применяя методы вариационного исчисления [54], решим гладкую экстремальную задачу

$$
\Lambda_{1}(y, x) \rightarrow \inf , \quad(y, x) \in \mathbb{R} \times \operatorname{Ran}\left(A_{w_{0}}\right) .
$$

Согласно лемме 3.6 при $0<p \leqslant 2$ выполнено неравенство $\|x\|_{p} \leqslant\|x\|_{2}$. Учитывая это неравенство, формулу (4.35) и первую теорему Вейерштрасса (см. замечание 9.1 в монографии [53]), заключаем, что минимум в задаче (4.38) достигается.

Используя формулы (4.8), (4.9) и (4.36), убеждаемся, что стационарные точки задачи (4.38) удовлетворяют следующей системе уравнений:

$$
\begin{aligned}
& \frac{\partial}{\partial y} \Lambda_{1}(y, x) \equiv \beta^{2} \int_{0}^{1} x(t) d t+\beta^{2} y-\frac{p \int_{0}^{1}|x(t)+y|^{p-1} \operatorname{sgn}(x(t)+y) d t}{\int_{0}^{1}|x(t)+y|^{p} d t}=0 \\
& \frac{\partial}{\partial x} \Lambda_{1}(y, x) \equiv \beta^{2} x(t)+\beta^{2} y-\frac{p|x(t)+y|^{p-1} \operatorname{sgn}(x(t)+y)}{\int_{0}^{1}|x(t)+y|^{p} d t}=0 .
\end{aligned}
$$

Несложно видеть, что указанная система уравнений имеет только два решения: $\pm z_{0}= \pm\left(y_{0}, 0\right)$. Учитывая формулы (4.10), (4.36) и неравенство Шварца, заключаем, что квадратичная форма, построенная по второй производной $\Lambda_{1}^{\prime \prime}\left(z_{0}\right)$, удовлетворяет соотношениям

$$
\left\langle z, \Lambda_{1}^{\prime \prime}\left(z_{0}\right) z\right\rangle_{B}=\beta^{2}(p-2)\left(\langle x, \mathbf{1}\rangle^{2}-\langle x, x\rangle\right)+2 \beta^{2}(y+\langle x, \mathbf{1}\rangle)^{2} \geqslant 0, \quad z=(y, x) .
$$


Таким образом, для точек $\pm z_{0}$ выполнены необходимые условия минимума первого и второго порядков [54]. Нетрудно найти, что

$$
\Lambda_{1}\left(z_{0}\right)=\Lambda_{1}\left(-z_{0}\right)=\frac{p}{2}-\frac{p}{2} \ln \frac{p}{\beta^{2}} .
$$

Учитывая вышеизложенное, в частности то, что минимум в задаче (4.38) достигается и существуют только две стационарные точки, убеждаемся, что второй минимум в (4.37) достигается только в точках $\pm\left(y_{0}, 0\right)$. Лемма доказана.

ДокАЗАТЕЛЬСтво лЕммы 4.2. Первое утверждение леммы 4.2 и формулы (4.3), (4.4) вытекают из лемм 4.3-4.7. Наличие всего двух точек минимума при $0<p \leqslant 2$ следует непосредственно из лемм 4.3-4.7. Существование порога $p_{0}(\beta)$ доказано в лемме 4.5. В лемме 4.6 доказано, что для точек $\pm z_{0}$ выполнены необходимые условия минимума первого и второго порядков при $2<p \leqslant p_{0}(\beta)$. Можно показать, что при $2<p \leqslant p_{0}(\beta)$ минимум в экстремальной задаче (4.2) также доставляют только две точки $\pm z_{0}$.

Используя свойства гладкости функционала $F$ и известный вид гильбертова пространства $H_{w_{0}}$, ассоциированного с ковариационным оператором $A_{w_{0}}$, несложно показать, что при $0<p \leqslant p_{0}(\beta)$

$$
\inf _{z \in H_{A_{\nu}}}\left\{F(z)+\frac{1}{2}\|z\|_{A_{\nu}}^{2}\right\}
$$

также достигается только в двух точках $\pm z_{0}$. Лемма доказана.

Лемма 4.8. Пусть биксированъц числа $\beta>0 u p>p_{0}(\beta)$. Тогда точки $\pm z_{0}$, заданные в (4.3), не являются точками локального минимума в экстремальной задаче (4.2).

ДокАЗАТЕЛьство. В самом деле, из леммы 4.5 и ее доказательства вытекает, что при $p>p_{0}(\beta)$ для стационарных точек $\pm z_{0}$ не выполнено необходимое условие минимума второго порядка. А именно, в силу формул (1.9), (4.15), (4.16) имеют место следующие соотношения при $p>p_{0}(\beta)$ :

$$
\left\langle e_{1}, \Lambda_{0}\left(z_{0}\right) e_{1}\right\rangle=\gamma_{1}=4 \pi^{2}+\beta^{2}(2-p)<0 .
$$

\section{5. ЗАВЕРШЕНИЕ ДОКАЗАТЕЛЬСТВА ТЕОРЕМЫ 1.1, ДОКАЗАТЕЛЬСТВО ПРЕДЛОЖЕНИЯ 1.3}

В силу лемм 4.1, 4.2 для модельного интеграла Лапласа $(3.24)$ при $0<p \leqslant p_{0}(\beta)$ выполнены условия 1-3 теоремы 2.1 , где следует положить $B=\mathbb{R} \times L^{\widetilde{p}}, P_{A}(d z)=$ $\nu_{\beta}(d z), f(x) \equiv 1, u=\sqrt{n}$ и взять функцию $F$ из (3.21). При этом у нас имеются всего две экстремальные точки $\pm z_{0}= \pm\left(y_{0}, 0\right)= \pm(\sqrt{p} / \beta, 0)$. Согласно формулам (4.1), (4.10)

$$
\begin{aligned}
\left\langle z, F^{\prime \prime}\left(z_{0}\right) z\right\rangle_{B} & =\left\langle z, F^{\prime \prime}\left(-z_{0}\right) z\right\rangle_{B}= \\
& =\beta^{2}(2-p)\langle x, x\rangle+\beta^{2} p\langle x, \mathbf{1}\rangle^{2}+4 \beta^{2} y\langle x, \mathbf{1}\rangle+\beta^{2} y^{2}
\end{aligned}
$$

где $z=(y, x)$. Таким образом, интегралы (2.1) для точек $\pm z_{0}$ совпадают, обозначим их общее значение через $D=D(p)$. В силу формул $(2.1),(3.5),(3.15),(3.16)$ и (5.1) 
имеют место равенства

$$
\begin{aligned}
& D(p)=\int_{B} e^{-\left\langle z, F^{\prime \prime}\left(z_{0}\right) z\right\rangle / 2} \nu_{\beta}(d z)=\frac{\beta}{\sqrt{2 \pi}} \int_{-\infty}^{\infty} d y \times \\
& \quad \times \int_{L^{2}} \exp \left\{-\beta^{2} y^{2}-\frac{\beta^{2}(2-p)}{2}\langle x, x\rangle-\frac{\beta^{2} p}{2}\langle x, \mathbf{1}\rangle^{2}-2 \beta^{2} y\langle x, \mathbf{1}\rangle\right\} P_{w_{0}}(d x) .
\end{aligned}
$$

Значения интеграла (5.2) при разных $p$ приведены в следующей лемме.

Лемма 5.1. Интеграл $D(p)$ принимает следующие значения:

$$
D(p)= \begin{cases}\frac{\beta \sqrt{2-p}}{2 \sqrt{2} \operatorname{sh}((\beta / 2) \sqrt{2-p})}, & 0<p<2, \\ \frac{1}{\sqrt{2}}, & p=2, \\ \frac{\beta \sqrt{p-2}}{2 \sqrt{2} \sin ((\beta / 2) \sqrt{p-2})}, & 2<p<p_{0}(\beta) .\end{cases}
$$

ДокАЗАТЕЛьство. Применяя теорему Фубини к интегралу (5.2), убеждаемся, что при $0<p<p_{0}(\beta)$ выполнено равенство

$$
D(p)=\frac{\beta}{\sqrt{2 \pi}} \int_{L^{2}} \exp \left\{-\frac{\beta^{2}(2-p)}{2}\langle x, x\rangle-\frac{\beta^{2} p}{2}\langle x, \mathbf{1}\rangle^{2}\right\} Q(x) P_{w_{0}}(d x),
$$

где

$$
Q(x):=\int_{-\infty}^{\infty} e^{-\beta^{2} y^{2}-2 \beta^{2} y\langle x, \mathbf{1}\rangle} d y, \quad x \in L^{2} .
$$

Используя формулу 2.3.15.11 из справочника [57], получаем равенство

$$
Q(x)=\frac{\sqrt{\pi}}{\beta} e^{\beta^{2}\langle x, \mathbf{1}\rangle^{2}}, \quad x \in L^{2} .
$$

Из соотношений $(5.4),(5.5)$ вытекает следующее равенство при $0<p<p_{0}(\beta)$ :

$$
D(p)=\frac{1}{\sqrt{2}} \int_{L^{2}} \exp \left\{\frac{\beta^{2}(p-2)}{2}\left(\langle x, x\rangle-\langle x, \mathbf{1}\rangle^{2}\right)\right\} P_{w_{0}}(d x) .
$$

Дальнейшее вычисление интеграла нужно проводить отдельно для каждого из трех случаев доказываемой леммы.

1. Пусть фиксировано число $0<p<2$. Вычислим интеграл (5.6), применяя предложение 2.1, где мы положим

$$
B=L^{2}, \quad P_{A}=P_{w_{0}}, \quad A=A_{w_{0}}, \quad C=\beta^{2}(p-2) I .
$$

Легко видеть, что оператор $A^{-1}-C \equiv A_{w_{0}}^{-1}+\beta^{2}(2-p) I$ является строго положительным, следовательно, в силу формулы (2.4) справедливо соотношение

$$
M=\int_{L^{2}} e^{\beta^{2}(p-2)\langle x, x\rangle / 2} P_{w_{0}}(d x)<\infty .
$$

Согласно предложению 2.1 и формулам (2.5), (5.7) вероятностная мера

$$
\gamma(d x)=\frac{1}{M} e^{\beta^{2}(p-2)\langle x, x\rangle / 2} P_{w_{0}}(d x)
$$


является гауссовской мерой на $L^{2}$ с нулевым средним и инъективным ковариационным оператором

$$
A_{\gamma}=\left(A_{w_{0}}^{-1}+\beta^{2}(2-p) I\right)^{-1} \equiv\left(I+\beta^{2}(2-p) A_{w_{0}}\right)^{-1} A_{w_{0}} .
$$

Учитывая формулы (5.6) и (5.9), убеждаемся, что при $0<p<2$ справедливо равенство

$$
D(p)=\frac{M}{\sqrt{2}} \int_{L^{2}} e^{\beta^{2}(2-p)\langle x, \mathbf{1}\rangle^{2} / 2} \gamma(d x) .
$$

Найдем значения постоянной $M$ и интеграла из формулы (5.11). Используя лемму 3.3 настоящей работы и формулу 6.2.1.6 из справочника [57], получаем

$$
\operatorname{det}\left(I+\beta^{2}(2-p) A_{w_{0}}\right)=\prod_{k=1}^{\infty}\left(1+\frac{\beta^{2}(2-p)}{\pi^{2} k^{2}}\right)=\frac{\operatorname{sh}(\beta \sqrt{2-p})}{\beta \sqrt{2-p}} .
$$

В силу п. 2 предложения 2.1 и формул (5.8), (5.12) выполнено равенство

$$
M=\left[\operatorname{det}\left(I+\beta^{2}(2-p) A_{w_{0}}\right)\right]^{-1 / 2}=\frac{\sqrt{\beta}(2-p)^{1 / 4}}{\sqrt{\operatorname{sh}(\beta \sqrt{2-p})}} .
$$

Для вычисления интеграла из формулы (5.11) найдем величину

$$
\sigma^{2}:=\left\langle\mathbf{1}, A_{\gamma} \mathbf{1}\right\rangle=\int_{L^{2}}\langle x, \mathbf{1}\rangle^{2} \gamma(d x)
$$

далее мы полагаем, что параметр $\sigma$ неотрицателен. Пусть

$$
h(t):=\left(A_{\gamma} \mathbf{1}\right)(t), \quad t \in[0,1] .
$$

Функция $h(t)$, очевидно, удовлетворяет уравнению $A_{\gamma}^{-1} h=\mathbf{1}$. Это уравнение в силу формулы (5.10) эквивалентно уравнению $A_{w_{0}}^{-1} h+\beta^{2}(2-p) h=\mathbf{1}$, которое согласно лемме 3.2 эквивалентно следующей неоднородной граничной задаче:

$$
\begin{aligned}
h^{\prime \prime}(t)+\beta^{2}(p-2) h(t)+1 & =0, \quad t \in[0,1], \\
h(0)=h(1) & =0 .
\end{aligned}
$$

В результате замены переменной

$$
f(t)=h(t)+\frac{1}{\beta^{2}(p-2)}, \quad t \in[0,1],
$$

задача (5.16) принимает вид

$$
\begin{gathered}
f^{\prime \prime}(t)+\beta^{2}(p-2) f(t)=0, \quad t \in[0,1], \\
f(0)=f(1)=\frac{1}{\beta^{2}(p-2)} .
\end{gathered}
$$

Общее решение дифференциального уравнения из (5.18) в случае $0<p<2$ хорошо известно (см., например, справочник [56]). Учтем замену (5.17), получим, что единственное решение задачи (5.16) имеет вид

$$
h(t)=\frac{1}{\beta^{2}(p-2)}\left(\operatorname{ch}(t \beta \sqrt{2-p})+\frac{1-\operatorname{ch}(\beta \sqrt{2-p})}{\operatorname{sh}(\beta \sqrt{2-p})} \operatorname{sh}(t \beta \sqrt{2-p})-1\right),
$$

где $t \in[0,1]$. 
Используя формулы (5.14), (5.15) и (5.19), получаем после несложных преобразований, что

$$
\sigma^{2}=\int_{0}^{1} h(t) d t=\frac{1}{\beta^{2}(2-p)}\left(1-\frac{2 \operatorname{th}((\beta / 2) \sqrt{2-p})}{\beta \sqrt{2-p}}\right) .
$$

Учитывая неравенство $0<\operatorname{th} x<x$, верное для всех $x>0$, выводим следующие оценки:

$$
0<\sigma^{2}<\frac{1}{\beta^{2}(2-p)}
$$

Отсюда вытекает неравенство

$$
\tau^{2}:=\frac{1}{\sigma^{2}}+\beta^{2}(p-2)>0 .
$$

Используя равенство

$$
\gamma\left\{x \in L^{2}:\langle x, \mathbf{1}\rangle<t\right\}=\frac{1}{\sqrt{2 \pi} \sigma} \int_{-\infty}^{t} e^{-s^{2} / 2 \sigma^{2}} d s, \quad t \in \mathbb{R},
$$

и формулы (5.11), (5.21), убеждаемся, что справедливы соотношения

$$
D(p)=\frac{M}{2 \sigma \sqrt{\pi}} \int_{-\infty}^{\infty} \exp \left\{-\frac{t^{2}}{2}\left(\beta^{2}(p-2)+\frac{1}{\sigma^{2}}\right)\right\} d t=\frac{M}{\sigma \tau \sqrt{2}} .
$$

Применим формулы $(5.20),(5.21)$, получим

$$
\sigma \tau=\frac{\sqrt{2 \operatorname{th}((\beta / 2) \sqrt{2-p})}}{\sqrt{\beta}(2-p)^{1 / 4}} .
$$

С учетом соотношений (5.13), (5.23), (5.24) получаем после несложных преобразований равенство (5.3) для $0<p<2$.

2. Равенство (5.3) в случае $p=2$ вытекает непосредственно из формулы (5.6).

3. Пусть теперь $2<p<2+4 \pi^{2} / \beta^{2}$. Здесь удобно выделить две возможности: случай $2<p<2+\pi^{2} / \beta^{2}$ и случай $2+\pi^{2} / \beta^{2} \leqslant p<2+4 \pi^{2} / \beta^{2}$. Рассмотрим их по отдельности.

Пусть фиксировано число $2<p<2+\pi^{2} / \beta^{2}$. Вычисление интеграла (5.6) здесь также основано на предложении 2.1 и проводится по той же схеме, что и в случае $0<p<2$. А именно, мы применяем предложение 2.1 с учетом определений (5.7). В силу леммы 3.3 для $0 \neq x \in \operatorname{Ran}\left(A_{w_{0}}\right)$ имеем неравенство $\left\langle x, A_{w_{0}}^{-1} x\right\rangle>\pi^{2}$. Из него вытекает, что при $2<p<2+\pi^{2} / \beta^{2}$ оператор $A^{-1}-C \equiv A_{w_{0}}^{-1}+\beta^{2}(2-p) I$ является строго положительным. Следовательно, в силу формулы (2.4) справедливо соотношение (5.8). Согласно предложению 2.1 и формулам (2.5), (5.7) вероятностная мера $\gamma(d x)$, введенная в $(5.9)$, является гауссовской мерой на $L^{2}$ с нулевым средним и инъективным ковариационным оператором $A_{\gamma}$, заданным в (5.10). Учитывая формулы (5.6) и (5.9), убеждаемся, что в рассматриваемом случае также справедливо равенство (5.11).

Найдем коэффициент $M$ и интеграл, входящие в формулу (5.11). Используя лемму 3.3 настоящей работы и формулу 6.2.1.6 из справочника [57], теперь получаем равенства

$$
\operatorname{det}\left(I+\beta^{2}(2-p) A_{w_{0}}\right)=\prod_{k=1}^{\infty}\left(1+\frac{\beta^{2}(2-p)}{\pi^{2} k^{2}}\right)=\frac{\sin (\beta \sqrt{p-2})}{\beta \sqrt{p-2}}
$$


Отсюда в силу п. 2 предложения 2.1 и соотношения (5.8) выполнены равенства

$$
M=\left[\operatorname{det}\left(I+\beta^{2}(2-p) A_{w_{0}}\right)\right]^{-1 / 2}=\frac{\sqrt{\beta}(p-2)^{1 / 4}}{\sqrt{\sin (\beta \sqrt{p-2})}} .
$$

Для вычисления интеграла из формулы (5.11) найдем

$$
\sigma_{1}^{2}:=\left\langle\mathbf{1}, A_{\gamma} \mathbf{1}\right\rangle=\int_{L^{2}}\langle x, \mathbf{1}\rangle^{2} \gamma(d x)
$$

Положим

$$
h_{1}(t):=\left(A_{\gamma} \mathbf{1}\right)(t), \quad t \in[0,1] .
$$

Функция $h_{1}(t)$, очевидно, удовлетворяет уравнению $A_{\gamma}^{-1} h=\mathbf{1}$. Это уравнение в силу формулы (5.10) эквивалентно уравнению $A_{w_{0}}^{-1} h+\beta^{2}(2-p) h=\mathbf{1}$, которое согласно лемме 3.2 эквивалентно неоднородной граничной задаче (5.16). В результате замены переменной (5.17) задача (5.16) принимает вид (5.18). Общее решение дифференциального уравнения из (5.18) при $p>2$ хорошо известно (см., например, справочник [56]). Учтем замену (5.17), получим, что единственное решение задачи (5.16) при $2<p<2+\pi^{2} / \beta^{2}$ имеет вид

$$
h_{1}(t)=\frac{1}{\beta^{2}(p-2)}\left(\cos (t \beta \sqrt{p-2})+\frac{1-\cos (\beta \sqrt{p-2})}{\sin (\beta \sqrt{p-2})} \sin (t \beta \sqrt{p-2})-1\right),
$$

где $t \in[0,1]$. Используя формулы (5.26)-(5.28), получаем после несложных преобразований, что

$$
\sigma_{1}^{2}=\int_{0}^{1} h_{1}(t) d t=\frac{1}{\beta^{2}(p-2)}\left(\frac{2 \operatorname{tg}((\beta / 2) \sqrt{p-2})}{\beta \sqrt{p-2}}-1\right) .
$$

Отсюда с учетом неравенства $0<x<\operatorname{tg} x$, верного для всех $0<x<\pi / 2$, заключаем, что $\sigma_{1}^{2}>0$. Введем параметр

$$
\tau_{1}^{2}:=\frac{1}{\sigma_{1}^{2}}+\beta^{2}(p-2)>0, \quad \tau_{1}>0 .
$$

Используя равенство (5.22) и формулу (5.11), убеждаемся, что справедливы соотношения

$$
D(p)=\frac{M}{2 \sigma_{1} \sqrt{\pi}} \int_{-\infty}^{\infty} \exp \left\{-\frac{t^{2}}{2}\left(\beta^{2}(p-2)+\frac{1}{\sigma_{1}^{2}}\right)\right\} d t=\frac{M}{\sigma_{1} \tau_{1} \sqrt{2}} .
$$

Применяя формулы (5.29), (5.30), получаем равенство

$$
\sigma_{1} \tau_{1}=\frac{\sqrt{2 \operatorname{tg}((\beta / 2) \sqrt{p-2})}}{\sqrt{\beta}(p-2)^{1 / 4}} .
$$

С учетом этого равенства, используя соотношения (5.25), (5.31), получаем после несложных преобразований равенство (5.3) для значений $2<p<2+\pi^{2} / \beta^{2}$.

Пусть теперь фиксировано число $2+\pi^{2} / \beta^{2} \leqslant p<2+4 \pi^{2} / \beta^{2}$. Метод доказательства, примененный выше, теперь уже нельзя использовать. В данном случае мы 
применим предложение 2.1 в иной, более общей форме непосредственно к интегралу (5.6). А именно, положим в предложении 2.1

$$
\begin{gathered}
B=L^{2}, \quad P_{A}=P_{w_{0}}, \quad A=A_{w_{0}}, \\
\langle x, C x\rangle \equiv \beta^{2}(p-2)\left(\langle x, x\rangle-\langle x, \mathbf{1}\rangle^{2}\right) .
\end{gathered}
$$

В силу п. 2 предложения 2.1 и формулы (5.6)

$$
D(p)=\frac{1}{\sqrt{2}}\left[\operatorname{det}\left(I-A_{w_{0}} C\right)\right]^{-1 / 2} .
$$

Рассуждая так же, как при доказательстве п. 2 леммы 4.5, можно показать, что собственные значения оператора $A_{w_{0}} C: L^{2} \rightarrow L^{2}$ образуют множество

$$
\left\{\alpha_{k}\right\}_{k=1}^{\infty} \cup\left\{\widetilde{\alpha}_{k}\right\}_{k=1}^{\infty}, \quad \alpha_{k}=\widetilde{\alpha}_{k}=\frac{\beta^{2}(p-2)}{4 k^{2} \pi^{2}}, \quad k=1,2, \ldots
$$

Применяя формулу 6.2.1.6 из справочника [57] и равенство (5.33), мы убеждаемся, что и при $2+\pi^{2} / \beta^{2} \leqslant p<2+4 \pi^{2} / \beta^{2}$ справедлива формула (5.3). Довольно длинные вычисления здесь опущены; стоит отметить, что описанный в последнем случае метод применим ко всем значениям $2<p<2+4 \pi^{2} / \beta^{2}$. Лемма доказана.

ДоКАЗАТЕЛЬСТВо ТЕОРЕмы 1.1. Согласно лемме 5.1 для модельного интеграла (3.24) при $0<p<p_{0}(\beta)$ выполнено также условие 4 теоремы 2.1. Учитывая этот факт, а также выводы, изложенные в начале настоящего раздела, заключаем, что к интегралу (3.24) применима теорема 2.1 , где следует положить $B=\mathbb{R} \times L^{\widetilde{p}}$, $P_{A}(d z)=\nu_{\beta}(d z), f(x) \equiv 1, u=\sqrt{n}, m=2$ и взять функцию $F$ из (3.21). С учетом формул $(2.2),(4.4),(5.2)$ получаем при $0<p<p_{0}(\beta)$ и $n \rightarrow \infty$ следующие соотношения:

$$
\begin{aligned}
K_{2}(n, \beta, p) & =2 e^{-n \Lambda\left(z_{0}\right)} D(p)(1+o(1))= \\
& =2 \exp \left\{-\frac{n p}{2}\left(1-\ln \frac{p}{\beta^{2}}\right)\right\} D(p)(1+o(1)) .
\end{aligned}
$$

Учитывая формулы (3.1), (3.23), получаем равенство

$$
b_{n}(\beta, p)=\frac{(-1)^{n} e^{\beta / 2}}{n ! \beta} \beta^{n(p+2) / 2} n^{n p / 2} K_{2}(n, \beta, p)
$$

для фиксированных чисел $p>0, \beta>0, n \geqslant 1$. Из формул (5.34), (5.35) вытекает следующее соотношение при $0<p<p_{0}(\beta)$ и $n \rightarrow \infty$ :

$$
b_{n}(\beta, p)=\frac{2(-1)^{n} e^{\beta / 2}}{n ! \beta} \beta^{n(2-p) / 2}\left(\frac{n p}{e}\right)^{n p / 2} D(p)(1+o(1)) .
$$

Отсюда, учитывая формулу (5.3), получаем соотношения (1.10)-(1.12). Теорема доказана.

ДОКАЗАТЕЛЬСТВО ПРЕДЛОЖЕНИЯ 1.3. При $p=p_{0}$ к интегралу (3.24) применима формула (2.3) теоремы 2.2 , где $B=\mathbb{R} \times L^{\widetilde{p}}, P_{A}(d z)=\nu_{\beta}(d z), u=\sqrt{n}$ и функция $F$ 
задана в (3.21). Используя формулы (2.3) и (4.4) при $p=p_{0}$, получаем следующие соотношения при $n \rightarrow \infty$ :

$$
\lim _{n \rightarrow \infty} \frac{1}{n} \ln K_{2}\left(n, \beta, p_{0}\right)=-\Lambda\left(z_{0}\right)=-\frac{p_{0}}{2}\left(1-\ln \frac{p_{0}}{\beta^{2}}\right) .
$$

Таким образом, применив равенство (5.35) при $p=p_{0}$, получаем соотношение (1.13). Предложение доказано.

Благодарности. Работа выполнена при поддержке РФФИ (грант № 11-01-00050).

\section{Список литературы}

[1] Дж. Глимм, А. Джаффе, Математические методы квантовой физики. Подход с использованием континуальных интегралов, Мир, М., 1984.

[2] Х.-О. Георги, Гиббсовские меры и фазовые переходы, Мир, М., 1992.

[3] В. И. Питербарг, В. Р. Фаталов, УМH, 50:6(306) (1995), 57-150.

[4] В. Р. Фаталов, ТМФ, 168:2 (2011), 299-340.

[5] М. Рид, Б. Саймон, Методы современной математической физики, т. 1: Функииональный анализ, Мир, М., 1977; т. 2: Гармонический анализ. Самосопряженность, Мир, М., 1978; т. 4: Анализ операторов, Мир, М., 1982.

[6] Р. Фейнман, "Пространственно-временной поход к нерелятивистской квантовой механике", Вопросъ причинности в квантовой механике, ИЛ, М., 1955, 167-207.

[7] В. Р. Фаталов, "О методе Лапласа для гауссовских мер в банаховом пространстве", ТВП (в печати).

[8] В. Р. Фаталов, Изв. РАН. Сер. матем., 75:4 (2011), 189-223.

[9] В. Р. Фаталов, ТВП, 53:1 (2008), 72-99.

[10] S. Albeverio, V. Fatalov, V. Piterbarg, J. Math. Sci. Univ. Tokyo, 16:1 (2009), 55-93.

[11] В. Р. Фаталов, Изв. РАН. Сер. матем., 75:4 (2011), 189-223.

[12] В.Р. Фаталов, Точные асимптотики вероятностных распределений и функииональных интегралов. Метод Лапласа, Дисс. ... докт. физ.-матем. наук, МГУ, М., 2009.

[13] B. Simon, Functional Integration and Quantum Physics, Pure and Applied Mathematics, 86, Academic Press, New York, 1979.

[14] W. Janke, A. Pelster (eds.), Path Integrals: New Trends and Perspectives, Proceedings of the 9th International Conference (Dresden, September 23-28, 2007), World Sci., Hackensack, NJ, 2008.

[15] J. Zinn-Justin, "Functional integrals in physics: the main achievements", Path Integrals: New Trends and Perspectives, Proceedings of the 9th International Conference (Dresden, September 23-28, 2007), eds. W. Janke, A. Pelster, World Sci., Hackensack, NJ, 2008, $251-260$.

[16] И. М. Ковальчик, Л. А. Янович, Обобщенный винеровский интеграл и некоторые его приложения, Наука и техника, Минск, 1989.

[17] R. S. Ellis, J. S. Rosen, Comm. Math. Phys., 82:2 (1981), 153-181.

[18] R. S. Ellis, J.S. Rosen, Trans. Amer. Math. Soc., 273:2 (1982), 447-481.

[19] C. M. Bender, T. T. Wu, Phys. Rev., 184 (1969), 1231-1260.

[20] C. M. Bender, T. T. Wu, Phys. Rev. Lett., 27:7 (1971), 461-465.

[21] C. M. Bender, T. T. Wu, Phys. Rev. D, 7 (1972), 1620-1636.

[22] T. Banks, C. M. Bender, T. T. Wu, Phys. Rev. D, 8:10 (1973), 3346-3366.

[23] C. M. Bender, T. T. Wu, Phys. Rev. Lett., 37:3 (1976), 117-120.

[24] Л. Н. Липатов, ЖКЭТФ, 72:2 (1972), 411-427.

[25] Л.Н. Липатов, Писъма в ЖКЭТФ, 25:2 (1977), 116-119. 
[26] E. Brézin, J. C. Le Guillou, J. Zinn-Justin, Phys. Rev. D, 15:6 (1977), 1544-1557.

[27] E. Brézin, J. C. Le Guillou, J. Zinn-Justin, Phys. Rev. D, 15:6 (1977), 1558-1564.

[28] J. C. Le Guillou, J. Zinn-Justin (eds.), Large Order Behaviour of Perturbation Theory, North-Holland, Amsterdam, 1990.

[29] Н. Н. Боголюбов, Д. В. Ширков, Введение в теорию квантованных полей, Наука, М., 1984 .

[30] В. В. Белокуров, Д. В. Ширков, Теория взаимодействия частии, Наука, М., 1986.

[31] В. В. Белокуров, Ю. П. Соловьев, Е. Т. Шавгулидзе, Метод построения квантовополевой теории возмущений со сходящимися рядами, Препринт НИИЯФ МГУ 95-31/395, МГУ, М., 1995.

[32] J. Zinn-Justin, Phys. Rep., 70:2 (1981), 109-167.

[33] С. Г. Крейн (ред.), Функииональный анализ, Наука, М., 1972.

[34] Б. Саймон, Модель $P(\varphi)_{2}$ эвклидовой квантовой теории поля, Мир, М., 1976.

[35] Т. Като, Теория возмущений линейных операторов, Мир, М., 1972.

[36] B. Simon, Ann. Phys., 58:1 (1970), 76-136.

[37] E. Lieb, Bull. Amer. Math. Soc., 82:5 (1976), 751-753.

[38] Н. Икэда, С. Ватанабэ, Стохастические дифференииальные уравнения и диффузионные процессы, Наука, М., 1986.

[39] Т. Хида, Броуновское движение, Наука, М., 1987.

[40] А.Н. Бородин, П. Салминен, Справочник по броуновскому движению, Лань, СПб., 2000.

[41] Х.-С. Го, Гауссовские меры в банаховых пространствах, Мир, М., 1979.

[42] Н. Н. Вахания, В. И. Тариеладзе, С. А. Чобанян, Вероятностные распределения в банаховых пространствах, Наука, М., 1985.

[43] М. А. Лифшиц, Гауссовские случайные функиии, ТВиМС, Киев, 1995.

[44] В. И. Богачев, Гауссовские меры, Физматлит, М., 1997.

[45] S. R. S. Varadhan, Comm. Pure Appl. Math., 19:3 (1996), 261-286.

[46] S. R. S. Varadhan, Lett. Math. Phys., 88:1-3 (2009), 175-185.

[47] E. Olivieri, M.E. Vares, Large Deviations and Metastability, Encyclopedia of Mathematics and its Applications, 100, Cambridge Univ. Press, Cambridge, 2005.

[48] R. S. Ellis, Entropy, Large Deviations, and Statistical Mechanics, Springer, Berlin, 2006.

[49] E. Bolthausen, Prob. Theory Relat. Fields, 72:2 (1986), 305-318.

[50] И. И. Гихман, А. В. Скороход, Теория случайных прочессов, т. 1, Наука, М., 1971.

[51] Н. Данфорд, Дж. Т. Шварц, Линейные операторь. Часть I. Общая теория, УРСС, M., 2004.

[52] М. А. Красносельский, П.П. Забрейко, Е. И. Пустыльник, П. Е. Соболевский, Интегральные операторы в пространствах суммируемых функиий, Наука, М., 1966.

[53] М. М. Вайнберг, Вариационный метод и метод монотонных операторов, Наука, М., 1972.

[54] В. М. Алексеев, В. М. Тихомиров, С. В. Фомин, Оптималъное управление, Наука, М., 1979.

[55] К. Ферник, "Регулярность траекторий гауссовских случайных функций”, Случайнъе прочессы. Выборочные функиии и пересечения, Математика. Новое в зарубежной науке. Вып. 10, ред. Ю. К. Беляев, Мир, М., 1978, 63-132.

[56] Э. Камке, Справочник по обыкновенным дифференциальным уравнениям, Наука, М., 1961.

[57] А.П. Прудников, Ю.А. Брычков, О.И. Маричев, Интеграль и рлды. Элементарные функиии, Наука, М., 1981. 